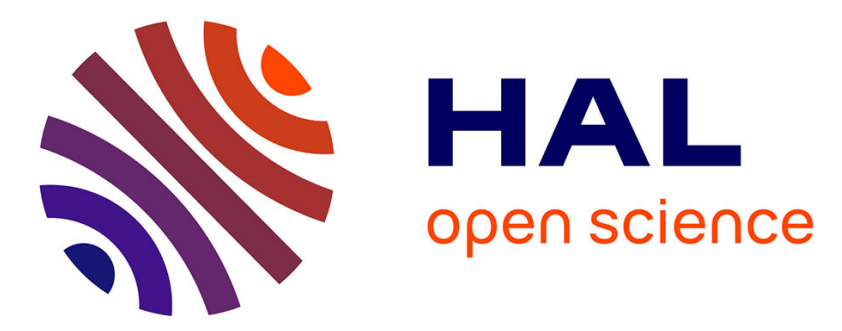

\title{
General Synthesis Method for Dispersively Coupled Resonator Filters With Cascaded Topologies
} Yan Zhang, Fabien Seyfert, Smain Amari, Martine Olivi, Ke-Li Wu

\section{To cite this version:}

Yan Zhang, Fabien Seyfert, Smain Amari, Martine Olivi, Ke-Li Wu. General Synthesis Method for Dispersively Coupled Resonator Filters With Cascaded Topologies. IEEE Transactions on Microwave Theory and Techniques, 2020, pp.15. 10.1109/TMTT.2020.3041223 . hal-03070731

\section{HAL Id: hal-03070731 https://hal.inria.fr/hal-03070731}

Submitted on 15 Dec 2020

HAL is a multi-disciplinary open access archive for the deposit and dissemination of scientific research documents, whether they are published or not. The documents may come from teaching and research institutions in France or abroad, or from public or private research centers.
L'archive ouverte pluridisciplinaire $\mathbf{H A L}$, est destinée au dépôt et à la diffusion de documents scientifiques de niveau recherche, publiés ou non, émanant des établissements d'enseignement et de recherche français ou étrangers, des laboratoires publics ou privés. 


\title{
General Synthesis Method for Dispersively Coupled Resonator Filters With Cascaded Topologies
}

\author{
Yan Zhang, Student Member, IEEE, Fabien Seyfert, Smain Amari, Martin Olivi and Ke-Li Wu, Fellow, IEEE
}

\begin{abstract}
This paper presents a new synthesis technique for circuits which may include dispersively coupled resonators and admitting an overall cascaded topology. A decomposition technique of Darlington type is first introduced to split the original response $S$ of the filter, taken as its scattering matrix, into $\mathrm{m}$ sub-responses $S^{1}, \ldots, S^{m}$ corresponding to each sub-block of the cascaded circuital structure. Each individual sub-response $S^{k}$ is then synthesized separately. In the second part of the paper, the state space equations governing the model of dispersively coupled resonators are detailed. An extension to the case of dispersive coupling of the shortest path rule, which determines the maximum number of finite TZs realizable by a given topology, is then introduced. Congruent transformations which extend the concept of rotations or similarity transformations while preserving the filter response are exploited to reduce the individual synthesis problems to the determination of a basis of vectors verifying certain orthogonality relations. A direct synthesis technique for dispersive building blocks like duplets, triplets and quadruplets, is then given in the form of an orthogonalization procedure used for the computation of the desired basis. This approach is then combined with the aforementioned decomposition technique to produce a versatile algorithm able to synthesise hybrid circuits made of cascaded sub-blocks of different orders and types that implement each a subset of the overall TZs by means of coupling topologies containing a mixture of dispersive and non-dispersive couplings. A first synthesis example is detailed where two dispersive duplets are combined with a classical quadruplet to realize a symmetric 6-4 response. A hardware implementation of the synthesized circuit is presented in combline technology. A second example proposes a slightly more involved coupling topology able to realize 10-8 asymmetric responses by means of 4 cascaded basic dispersive blocks.
\end{abstract}

Index Terms - Congruent transformation, Coupling Matrix, Dispersive couplings, Filter synthesis, Microwave filters

\section{INTRODUCTION}

$\mathbf{T}$ RANSMISSION zeros (TZs) at finite frequencies are critical characteristics of modern microwave filters where they are used to improve the out-of-band rejection to satisfy the stringent specifications of communication systems. Traditionally, two different techniques are used to generate them. The first one involves splitting an incoming signal into two or more signals which are then recombined at another point or node down the structure. A TZ results whenever the combined signals interfere destructively at the combining

Manuscript received July 4, 2020; revised October 5, 2020. The work described in this paper was partially supported by the Hong Kong Ph.D Fellowship. (Corresponding author: Fabien Seyfert)

Yan Zhang and Ke-Li Wu are with the Department of Electronic Engineering, The Chinese University of Hong Kong, Shatin, Hong Kong (e-mail: yzhang@link.cuhk.edu.hk; klwu@cuhk.edu.hk). Fabien Seyfert and Matine Olivi are with INRIA, Sophia-Antipolis, France (e-mail: fabien.seyfert@inria.fr, martine.olivi@inria.fr), Smain Amari is with the Royal Military College of Canada, Kingston, Canada (e-mail: smain.amari@rmc.ca). node. The number and locations in the complex plane of TZs thus generated depend on the details of the structure between the splitting and recombining points. The technique is widely used in industry in the form of cross-coupled topologies where two or more (less frequently) signal paths are provided by coupling non-adjacent resonators [1]. Topologies ranging from triplets and quadruplets to extended box and N-tuplets have been reported [2], [3]. Cascaded triplets and cascaded quadruplets [3] are preferred by the industry mainly because they are canonical [4] easy to realize and control their own TZs.

The second technique consists in using a shunt element which short-circuits the main and only signal path to ground at the finite frequency of the TZ. The shunt element is usually a dangling resonator in a bandstop configuration. Phase shifts or non-resonating nodes are introduced to control the passband of the filter (matching) [5]. Only TZs on the imaginary axis of the complex plane can be generated by this technique. It has the advantage of generating and completely controlling one TZ by a dedicated dangling element. More recently, dispersive coupling in which the coupling coefficients vary linearly with the normalized low-pass frequency was shown to produce additional TZs even in all-pole topologies such as in-line configurations. Each dispersive coupling coefficient contains a constant term and a term that varies linearly with the frequency. The mechanism behind the generation of TZs within this technique can be viewed as a combination of the previous two. The constant term and the linear term provide two separate signal paths. A TZ results when the signals of the two paths are $180^{\circ}$ out of phase but equal in magnitude. In other words, at the frequency of the TZ, the dispersive coupling completely blocks the signal by opening the circuit. Such a TZ is completely controlled by the dispersive coupling that generates it as long as it is the only path for the signal. Dispersive coupling can also be used in cross-coupled topologies where it provides the phase diversity that is needed to bring about destructive interference. It no longer controls the TZ by itself in this case. For example, a quadruplet in which the coupling coefficient between resonators 1 and 4 is dispersive is able to produce three TZs instead of only two when all the coupling coefficients are assumed constant. In this case, the dispersive coupling, although instrumental in causing a third TZ, does not control any of the TZs by itself.

In this paper we first introduce a decomposition method adapted to the synthesis of circuits with cascaded topologies. These topologies are characterised by a set of $m$ subtopologies successively chained to one another in such a manner that the output resonator of sub-topology $k$ is also the 
input resonator of sub-topology $k+1$. In other words, any two adjacent sub-circuits in the chain share exactly one resonator which we call a pivot. The decomposition procedure which is of Darlington type operates on the overall $2 \times 2$ response $S$ of the filter and furnishes $m$ sub-response $S^{k}$ that when properly combined will reproduce the original scattering matrix $S$. By essence in Darlington type approaches the decomposition is characterised by a particular distribution of the TZs among the $m$ sub-responses $S^{k}$. This distribution will be chosen here according to the number of TZs that each sub-circuit can accommodate. The individual scattering matrices $S^{k}$ will then be synthesized according to the topology retained for each sub-block. These sub-blocks are eventually cascaded to obtain the overall filter.

We will then turn to an in-depth analysis of the dispersive coupled-resonator model. Microwave filters with dispersive coupling have attracted the attention of many researchers over the last decade [6]-[10]. They may be modeled by a coupling matrix of the form Mo $+\mathbf{M d} \Omega$ as was first proposed in [11], with Mo being the coupling matrix at the center frequency, Md being the slope coefficient matrix, and $\Omega$ being the normalized frequency. The synthesis of these two coupling matrices has been reported in [12]-[22].

One group of dispersive synthesis method is by optimization. In [12] and [13], a zero-pole optimization is proposed to find Mo together with Md such that the resultant zeros and poles are forced to coincide with those of the synthesized filtering function. With the same optimization method, an adhoc dimensional design method for the filter configuration with stubs was proposed in [18] by considering the frequencydependence of the impedance or admittance matrices. This zero-pole optimization method is further improved in [14] to synthesize a coupling matrix with a dispersive source-load coupling, which corresponds to a response with more TZs than poles. Similar ideas are used in [23] but with a different cost function involving residues of the targeted impedance function. Projected gradient descent based on the transformation in [11] is utilized in [15] and further improved in [16] with a preconditioner to find Mo with a given Md. Very recently, a direct synthesis and design method by iteratively updating the characteristic polynomials has been proposed, being able to deal with nonlinear dispersion effects, an Mo can be found with a given $\operatorname{Md}(\Omega)$ and further guide the design of an inline waveguide filter [17].

The other kind of dispersive synthesis method is by analytic transformation. A transformation strategy was first proposed in [19] to transform the frequency-independent coupling matrix in Lattice topology to a designated coupling topology with localized frequency-dependent couplings. This method is improved in [20] and [21] to find a frequency-dependent coupling matrix in an inline topology. In [22], a similar method derives the transformation to find the dispersive coupling matrices for some other specific topologies. However, all these methods are based on lengthy rotation and scaling and with a limited type of topologies.

It is worth noting that repeated application of rotation and scaling is equivalent to a congruence transformation, which is similar to a similarity transformation but with the

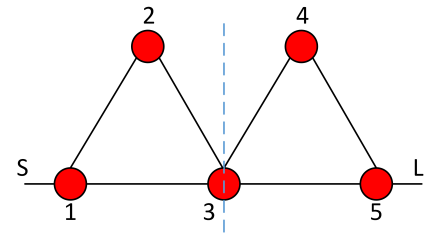

(a)

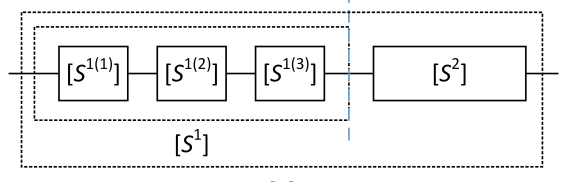

[S]

(b)

Fig. 1. (a) Cascaded topology made of two triplets. (b) Cascaded scattering parameters of sub-topologies and elementary sections.

transformation matrix being nonsingular instead of orthogonal [16]. This means that dispersive coupling matrices in different topologies can be obtained by finding the relevant congruence transformation: a direct approach to do so by means of orthogonalization procedures will be presented for different building blocks like dispersive duplets, triplets and quadruplets. We will also extend the shortest path rule given in [24], a crucial tool for the design of pertinent coupling topologies [25], to the dispersive setting.

Eventually, using our decomposition technique presented in the first part of the paper we will demonstrate how the synthesis of a broad range of cascaded topologies, including dispersive or/and non-dispersive sub-topolgies, can be handled. In particular, the canonical $n-1$ inline dispersive topology of [21] appears here as a cascaded sequence of dispersive duplets that can be synthesized independently in an elementary way. We illustrate the versatility of the new synthesis method at hand of the realization of a 6-pole combline filter, implementing a mixed cascaded topology composed of two dispersive duplets and a classical non-dispersive quadruplet. The numerical robustness and flexibility of the proposed method is further validated by the synthesis of a 10-pole filter with an involved cascaded topology.

\section{Divide AND Conquer Strategy}

We consider a lossless $2 \times 2$ scattering matrix $S$. Transmission zeros (TZs) are classically defined as the closed righthalf plane zeros of the rational function $S_{12} S_{21}$ [26]. The degree, or multiplicity of TZs belonging to the open righthalf plane is counted as usual for zeros of analytic functions or polynomials, whereas the multiplicity of TZs on the imaginary axis is counted as half the value of the classical definition. We denote by $\#(z, S)$ the degree of a TZ of $S$ located at $z$.

\section{A. Responses of Cascaded Topologies}

In this work we consider cascaded topologies where each of the $m$ sub-topologies has an identified input resonator and an output resonator. This excludes, for example, sub-topologies with several source to resonator couplings or source to load 


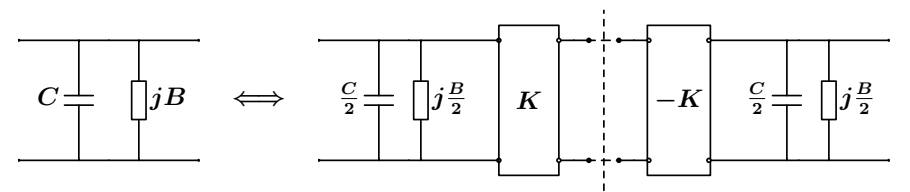

Fig. 2. Splitting a low-pass resonator and introducing two new ports.

couplings. Fig. 1(a) shows a cascaded topology made of two sub-topologies, triplets here, and where the common resonator is resonator 3 (pivot). We will first show that the $2 \times 2$ scattering matrix $S$ of a filter with a cascaded topology can be decomposed as,

$$
S=S^{1} \circ S^{2} \ldots S^{m}
$$

where each $S^{k}$ is a $2 \times 2$ filter response compatible with the $k^{t h}$ sub-topology. Here $S^{k} \circ S^{k+1}$ represents the scattering matrix obtained when port 2 of $S^{k}$ is chained to port 1 of $S^{k+1}$ [27]. In order to obtain the decomposition (1), we simply split the pivot, or common resonator, between sub-topologies $k$ and $k+1$, into two artificial cascaded inverters with opposite values as indicated in Fig. 2. This introduces $2 \times(m-1)$ new ports to be added to the filter besides the original input and output ports. Defining $S^{k}$ as the scattering parameter between two successive such ports immediately yields (1). After proper rescaling of its input and output resonators, the circuit corresponding to $S^{k}$ can again be represented by a classical coupling matrix. It should be noted that the splitting procedure of the common resonator is rather arbitrary: in Fig. 2 we chose a factor $\frac{1}{2}$, in order to split the capacitor and self coupling $j B$. Any other factor $1>\alpha>0$ would also work and result in another distribution of the common resonator between cascaded sections.

The application of decomposition (1) to the cascaded topology of Fig. 1 (a) leads to $S=S^{1} \circ S^{2}$, where $S^{1}$ and $S^{2}$ are order 3 responses, each of which can be implemented with a single finite TZ and two TZs at infinity: we will eventually see that $S^{1}$ and $S^{2}$ can be in turn split into three elementary sub-sections implementing each a particular TZ (see Fig. 1(b)). Note here that the cascade of two scattering matrices each of degree 3 results in a scattering matrix of order 5. This non-additive behaviour of degrees, is well explained by the circuital equivalence of Fig. 2: the connection of two resonators results here in a single common resonator but not two independent ones. On the functional side this decrease in degree is explained by the fact that $S^{1}$ and $S^{2}$ both have a TZ at infinity and so that at their common port

$$
S_{2,2}^{1}(\infty)=1=\overline{S_{1,1}^{2}(\infty)}
$$

where $\bar{x}$ means complex conjugate of $x$. The degree drop can be observed from the elementary chaining formula for lossless scattering parameters [27]

$$
S_{1,1}=S_{1,1}^{1}+\frac{S_{1,2}^{1} S_{2,1}^{1} S_{1,1}^{2}}{1-S_{2,2}^{1} S_{1,1}^{2}}=\frac{\operatorname{det}\left(S^{1}\right)\left(\left(S_{2,2}^{1}\right)^{*}-S_{1,1}^{2}\right)}{1-S_{2,2}^{1} S_{1,1}^{2}}
$$

where leading terms cancel each other out in the numerator and denominator polynomials in the last expression of (3) if
(2) holds. Recall that the scattering parameters are rational functions of the complex frequency.

It is now obvious that we need a rigorous method to determine the order of a scattering matrix that results from cascading two individual scattering matrices whose orders are known. This is summarized by the following mathematical property that governs the inter-connection of lossless $S$ matrices.

Proposition 2.1: Let $F$ and $G$ be two lossless $2 \times 2$ rational matrices chained such that port 2 of $F$ is connected to port 1 of $G$ and resulting in the matrix $E=F \circ G$. We denote by $H$ the set of transmission zeros that are common to $F$ and $G$ and where amatching type condition similar to (2) holds,

$$
\begin{array}{r}
H=\left\{z \in j \mathbb{R} \cup\{\infty\}, F_{2,2}(z)=\overline{G_{1,1}(z)}\right. \\
\text { and } \left.\left|F_{2,2}(z)\right|=\left|G_{2,2}(z)\right|=1\right\} .
\end{array}
$$

We have,

$$
\operatorname{deg}(E)=\operatorname{deg}(F)+\operatorname{deg}(G)-\operatorname{Card}(H)
$$

where $\operatorname{Card}(H)$ stands for cardinal number of the set $H$, i.e., the number of elements in it. When a TZ of $E$ is in $H$ then it is also a $\mathrm{TZ}$ of $F$ and $G$ and we say that it is shared via the chaining operation $F \circ G$, with both responses possessing a partial version of it. Physically, this means that around an element of $H$ both $F$ and $G$ behave similarly, i.e, each one completely reflects the signal. It, therefore, should not be surprising that the order of the combined scattering matrix is not the sum of the orders of the individual scattering matrices. However, the necessity of the conjugate matching condition in (4) which will eventually yield a zero-pole cancellation in (3) at the TZ is not obvious from this simple physical picture. The need for a rigorous mathematical formulation can be hardly over-emphasised here. A similar conclusion regarding the multiplicity of TZs should hold. For example, if two blocks have a first order TZ at the same location, what is the order of the TZ that the cascaded blocks generate at $z$ ? Since each block generates and controls its own $\mathrm{TZ}$ at $z$, we intuitively expect an overall $\mathrm{TZ}$ of second order. However, this is not always correct. It depends on the characteristics of the TZ. Once again, a the need of a rigorous mathematical rule is obvious. Indeed we have,

$$
\#(z, E)=\#(z, F)+\#(z, G)-\mathbb{1}_{H}(z)
$$

where $\mathbb{1}_{H(z)}$ is the indicator function of the set $H$. It is equal to 1 if $z$ is in $H$ and 0 otherwise. The rule says that the order of an overall $\mathrm{TZ}$ that is common to two cascaded blocks is the sum of the orders of the common TZ of each block unless the blocks satisfy the conditions in (4) in which case it is the sum of the orders reduced by one. For example, if blocks $F$ and $G$ generate a first order $\mathrm{TZ}$ at $z$ each, the $\mathrm{TZ}$ generated at $z$ when they are cascaded can be of order one or two depending on whether the conditions in (4) are satisfied or not. If they are satisfied, the overall order is one, if not it is two.

Assume all sub-topologies have at least one TZ at infinity (no Source-Load coupling) and therefore all filters responses $S^{k}$ are equal to the identity matrix $I d$ at infinity. One $\mathrm{TZ}$ at infinity is therefore shared in every of the $m-1$ chaining 


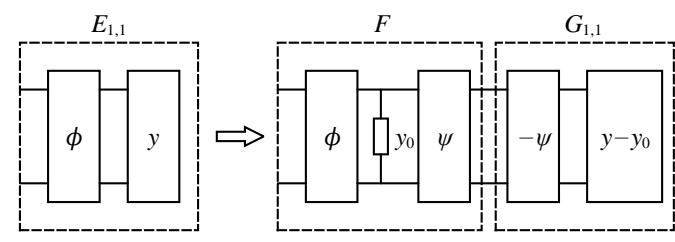

Fig. 3. Extraction of an elementary section from the overall response.

operation considered in (1). Under the generic simplifying hypothesis that none of the finite TZs $z_{i}$ of $S$ is shared among $\left(S^{k}\right)^{\prime} s$, the decomposition (1) translates as follow in terms of TZ multiplicities:

$$
\left\{\begin{aligned}
\#(\infty, S) & =\sum_{k=1}^{m} \#\left(\infty, S^{k}\right)-(m-1) \\
& =\sum_{k=1}^{m}\left(\frac{1}{m}+\left(\#\left(\infty, S^{k}\right)-1\right)\right) \\
\#\left(z_{i}, S\right) & =\sum_{k=1}^{m} \#\left(z_{i}, S^{k}\right)
\end{aligned}\right.
$$

Concretely, one infinite TZ of $S$ is shared among all the $S^{k}$ (7), while all other infinite or finite TZs need to be distributed among the $\left(S^{k}\right)^{\prime} s$ according to the limitations of each subtopology. For example, in our two triplet topology we start with a response $S$ of order 5 with two finite TZs $z_{1}, z_{2}$ and 3 TZs at infinity. One TZ at infinity is "shared" by $S_{1}$ and $S_{2}$, while two remaining infinite TZs and the finite TZs $z_{1}, z_{2}$ need to be distributed among each triplet. Each triplet can only accommodate one finite TZ, resulting in two possible decomposition of type (1) depending on which triplet implements which finite TZ.

\section{B. Extraction of Elementary scattering sections}

We have shown that the response $S$ of a circuit with cascaded topologies admits a functional decomposition of type (1), which is conceptually connected with the distribution of TZs among sub-topologies. We will now reverse the reasoning by showing that such a functional decomposition can be computed directly at start from $S$. Practically each $S^{k}$ in (1) will be obtained by extraction of several elementary sections with each associated to a particular TZ of the $k^{\text {th }}$ section (see Fig. 1(b)). Eventually, the circuit synthesis of each subresponse $S^{k}$ will yield the synthesis of $S$ by cascading circuits as in Fig. 2 (on the Figure from right to left this time).

Suppose that $E$ is a loss-less reciprocal two port of degree $n$ having a TZ $z_{0}=j \omega_{0}$ with value

$$
\gamma=E_{1,1}\left(z_{0}\right) \text {, and }|\gamma|=1 \text {. }
$$

Let us consider the problem of extracting from $E$ an elementary scattering matrix $F$ of McMillan degree 1 with a $\mathrm{TZ} z_{0}=j \omega_{0}$. The scattering matrix of the remaining section is denoted by $G$. This means that we need to show that $E$ can be written as $E=F \circ G$ with $G$ a loss-less two port of degree less or equal to $n$.
From the first part of the chaining equation (3) we intuitively expect two distinct situations. Either

$$
F_{2,2}\left(z_{0}\right) \neq \overline{G_{1,1}\left(z_{0}\right)}
$$

in which case the value and the derivative of $F_{1,1}$ should coincide with those of $E_{1,1}$ at $z_{0}$ as a consequence of the double zero present at $z_{0}$ in the term

$$
\frac{F_{1,2} F_{2,1} G_{1,1}}{1-F_{2,2} G_{1,1}}
$$

Or when the matching condition between $F$ and $G$ holds, that is $1-F_{22} G_{22}$ has a simple zero at $z_{0}$, we still have $F_{1,1}\left(z_{0}\right)=\gamma$ while the status of the derivative $F^{\prime}\left(z_{0}\right)$ becomes uncertain because of the pole-zero cancellation occurring in (9).

We use here an abstract circuital procedure to determine the scattering matrix $F$ : the latter is completely general and independent of the final circuit chosen to implement the filter. We first de-embed an ideal phase shifter with constant phase $\phi$ such that $e^{-j 2 \phi}=-\gamma$ in Fig. 3. This is always possible given that $|\gamma|=1$. The reflection at port 1 of the remaining two port is then $-E_{1,1} / \gamma$. We then compute its input admittance:

$$
y=\frac{1-\left(-E_{1,1} / \gamma\right)}{1+\left(-E_{1,1} / \gamma\right)}=\frac{1+E_{1,1} / \gamma}{1-E_{1,1} / \gamma}
$$

The positive real function $y$ has a simple pole at $z_{0}$, whose residue $r$ is therefore positive and explicitly computed to be

$$
r=\frac{1+E_{1,1}\left(z_{0}\right) / \gamma}{-E_{1,1}^{\prime}\left(z_{0}\right) / \gamma}=\frac{-2}{E_{1,1}^{\prime}\left(z_{0}\right) / E_{1,1}\left(z_{0}\right)} .
$$

Although not obvious at this point, the logarithmic derivative in this equation is indeed real and negative, as will be shown later.

Now following Darlington's procedure, a parallel admittance $y_{0}$ with a single and simple pole at $z_{0}$ and a positive residue $r_{0}$ can be extracted from $y$ as long as $r_{0} \leq r$. Coming back to the scattering domain and forming the two-port $F$ as in Fig. 3, we have:

$$
F_{1,1}\left(z_{0}\right)=\gamma, \frac{F_{1,1}^{\prime}\left(z_{0}\right)}{F_{1,1}\left(z_{0}\right)} \leq \frac{E_{1,1}^{\prime}\left(z_{0}\right)}{E_{1,1}\left(z_{0}\right)} .
$$

where the last inequality is a direct reformulation of the condition $r_{0} \leq r$. The second phase shifter with phase $\psi$ (see Fig. 3) whose introduction is completely transparent for the extraction process has been introduced here in order to adjust the phase of the extracted section. We have therefore proven that $E_{1,1}=F \circ G_{1,1}$, from which it is straightforward to show that there exists a loss-less two port $G$ (with $G_{1,1}$ defined as in Fig. 3), obtained by de-chaining the reciprocal two port $F$ from $E$, and such that $E=F \circ G$.

Because of its importance in the context of extraction, mathematicians have given the name "angular derivative" [28][chap. 21] to the quantity $E^{\prime} / E$ evaluated at an extremal point $z_{0}$ on the imaginary axis where $\left|E_{1,1}\left(z_{0}\right)\right|=1$. We will denote,

$$
\operatorname{Ang}\left(E_{1,1}\right)\left[z_{0}\right]=E_{1,1}^{\prime}\left(z_{0}\right) / E_{1,1}\left(z_{0}\right) .
$$

From the expansion

$$
E_{1,1}\left(z_{0}+s\right)=E_{1,1}\left(z_{0}\right)\left(1+E_{1,1}^{\prime}\left(z_{0}\right) / E_{1,1}\left(z_{0}\right)\right) s+o(s)
$$


with $s=j \omega$ being the variable of frequency, it is immediately seen that the angular derivative is real and $\operatorname{Ang}\left(E_{1,1}\right)\left[z_{0}\right]<$ 0 as otherwise the modulus of $E_{1,1}$ would exceed 1 in the vicinity of $z_{0}$ in the right half plane. Note that this is also coherent with the positivity of the residue $r$ found in (10). If $E_{1,1}$ has an extremal point at infinity the angular derivative is defined as the limiting value obtained in (12) at $s^{\prime}=0$ after the change in variable $s^{\prime}=1 / s$. If $E_{1,1}=p / q$ is of degree $n$ we have

$$
\operatorname{Ang}\left(E_{1,1}\right)[\infty]=\frac{p_{n-1}}{p_{n}}-\frac{q_{n-1}}{q_{n}}
$$

where $p_{k}$ denotes the coefficient in $s^{k}$ of the polynomial $p$.

Coming back to our extracted section $F$ we distinguish as expected between two situations. In the case $r_{0}=r$, that is

$$
\operatorname{Ang}\left(F_{1,1}\right)\left[z_{0}\right]=\operatorname{Ang}\left(E_{1,1}\right)\left[z_{0}\right]
$$

we say that $F$ realizes an entire extraction at $z_{0}$. In particular $\operatorname{deg}(G)=\operatorname{deg}(E)-1$ holds as well as $1-F_{2,2}\left(z_{0}\right) G_{1,1}\left(z_{0}\right) \neq$ 0 because no pole is present anymore in the admittance $y-y_{0}$ at $z_{0}$.

When $r_{0}<r$ holds, that is

$$
\operatorname{Ang}\left(F_{1,1}\right)\left[z_{0}\right]<\operatorname{Ang}\left(E_{1,1}\right)\left[z_{0}\right]
$$

we speak of partial extractions, as $y-y_{0}$ still contains a portion of the pole at $z_{0}$. In this case it is readily verified that $\operatorname{deg}(G)=\operatorname{deg}(E)$ and that

$$
F_{2,2}\left(z_{0}\right)=\overline{G_{1,1}\left(z_{0}\right)},
$$

which is the matching condition mentioned at $\infty$ in (2) and in the general case in definition of the set $H$ in (4).

We now give explicit expressions for degree one sections tailored for the extraction of TZs at infinity and finite frequency and that will serve for the computation of the $S_{k}^{\prime} s$ in (1).

1) Extraction of a $T Z$ at $\infty$ : Define

$$
\mathcal{L}\left(\infty, \gamma, \zeta_{0}\right)[s]=\frac{1}{s-\zeta_{0}}\left[\begin{array}{cc}
\gamma s & \sqrt{\gamma} \zeta_{0} \\
\sqrt{\gamma} \zeta_{0} & s
\end{array}\right]
$$

which is an elementary lossless section with a TZ at infinity with value $\gamma$ and angular derivative $\zeta_{0}$ (the value of a TZ is the value of coefficient $(1,1)$ at the TZ's location). If $E$ is a lossless response with a TZ at infinity with value $\gamma$ and angular derivative $\zeta$, then $\mathcal{L}\left(\infty, \gamma, \zeta_{0}\right)$ can be extracted from it provided $\zeta_{0} \leq \zeta$. This means that there exists $G$ lossless, such that $E=\mathcal{L}\left(\infty, \gamma, \zeta_{0}\right) \circ G$. If $\zeta_{0}=\zeta$, the extraction is entire and $\operatorname{deg}(G)=\operatorname{deg}(E)-1$, otherwise the extraction is partial, $\operatorname{deg}(G)=\operatorname{deg}(E)$ and $G_{1,1}(\infty)=1=\mathcal{L}\left(\infty, \gamma, \zeta_{0}\right)_{2,2}[\infty]$.

2) Extraction of $T Z$ at $z_{0}=j \omega_{0}$ : Define

$$
\mathcal{L}\left(z_{0}, \gamma, \zeta_{0}\right)[s]=\frac{1}{s-z_{0}-1 / \zeta_{0}}\left[\begin{array}{cc}
-\gamma / \zeta_{0} & s-z_{0} \\
s-z_{0} & -1 /\left(\gamma \zeta_{0}\right)
\end{array}\right]
$$

which is an elementary lossless section with a $\mathrm{TZ}$ at $z_{0}$ with value $\gamma$ and angular derivative $\zeta_{0}$ (for the reflection coefficient $(1,1)$ ). If $E$ is a lossless response with a $\mathrm{TZ}$ at $z_{0}$ with value $\gamma$ and angular derivative $\zeta$, then $\mathcal{L}\left(z_{0}, \gamma, \zeta_{0}\right)$ can be extracted from it provided $\zeta_{0} \leq \zeta$. This means that there exists $G$ lossless, such that $E=\mathcal{L}\left(\infty, \gamma, \zeta_{0}\right) \circ G$. If $\zeta_{0}=\zeta$, the extraction is entire and $\operatorname{deg}(G)=\operatorname{deg}(E)-1$, while otherwise the extraction is partial, $\operatorname{deg}(G)=\operatorname{deg}(E)$ and $G_{1,1}(\infty)=\gamma$. Note also that in any case the value at infinity is conserved, that is $E(\infty)=G(\infty)$.

3) Extraction of a complex TZ pair : In a perfect lossless and reciprocal setting, a complex TZ $z_{0}=\sigma+j \omega_{0}(\sigma>0)$ has necessarily an even multiplicity, 2 , in the elementary case, and this is because it is simultaneously a root of $S_{12}$ and $S_{21}$. One possible choice for its extraction is therefore to extract successively two elementary lossless, but non-reciprocal section of degree one, that will eventually form a reciprocal section when cascaded. We will here follow a different path which puts reciprocity on the foreground and ensures losslessness at the end of the extraction process. Although the angular derivative is usually only defined for imaginary TZs and in the lossless case we extend its definition to complex $z_{0}$ by using (12).

Say $S$ is a lossless reciprocal response of degree $n$ with a complex TZ $z_{0}$ and set as usual $\gamma=S_{1,1}\left(z_{0}\right)$ and $\zeta=$ $\operatorname{Ang}\left(S_{11}\right)\left[z_{0}\right]$. There exists $G$ of degree $n-1$, reciprocal but not necessarily lossless, such that $S=\mathcal{L}\left(z_{0}, \gamma, \zeta\right) \circ G$. We then perform a second extraction at the mirror value

$$
z_{0}^{\prime}=-\sigma+j \omega_{0}
$$

which is also a root of $E_{1,2}$ and $E_{2,1}$. Define $\gamma^{\prime}=G_{1,1}\left(z_{0}^{\prime}\right)$ and $\zeta^{\prime}=\operatorname{Ang}\left(G_{11}\right)\left[z_{0}^{\prime}\right]$ : there exists $G^{\prime}$ of degree $n-2$ such that $G=\mathcal{L}\left(z_{0}^{\prime}, \gamma^{\prime}, \zeta^{\prime}\right) \circ G^{\prime}$. The total extracted section, that is

$$
\mathcal{L}\left(z_{0}, \gamma, \zeta\right) \circ \mathcal{L}\left(z_{0}^{\prime}, \gamma^{\prime}, \zeta^{\prime}\right)
$$

is lossless and reciprocal. This process is the exact analog, on a functional level, of the sequential extraction of complex TZs by a quadruplet described in [3], where a complex matrix is obtained as an intermediary step. Also note that this process paves the way to the extraction of single complex TZs which are encountered within lossy responses.

4) Implementation techniques: Extraction of elementary sections is classical since Darlington's work on lossless synthesis [26], [29] as well as Youla's on broadband matching [30]. Practically one can use chain matrices in the impedance domain or T-matrices in the scattering setting to simplify chaining operations. We chose to work with T-matrices (expressing power waves at access 1 in terms of those at access 2 ) whose definition we briefly recall,

$$
\mathcal{T}(S)=\frac{1}{S_{2,1}}\left[\begin{array}{cc}
1 & -S_{2,2} \\
S_{1,1} & -\operatorname{det}(S)
\end{array}\right]
$$

The matrix $\mathcal{T}(S)$ has the same McMillan degree as $S$. Therefore simple polynomial expressions for the T-matrices of any of the degree one elementary sections we introduced can be obtained. This is generally true for any lossless response $S$ given in its polynomial Belevitch form [26]. The main interest of T-matrices is summarized by the following equation,

$$
\mathcal{T}\left(S_{1} \circ S_{2}\right)=\mathcal{T}\left(S_{1}\right) . \mathcal{T}\left(S_{2}\right)
$$

which transforms the chaining operation into a usual matrix multiplication. The extraction procedure is therefore cast to the inversion of a rational T-matrix which is particularly simple 


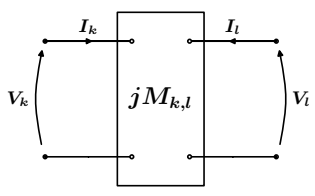

Fig. 4. A general two port coupling component.

in the case of reciprocal responses for which $\operatorname{det}(\mathcal{T}(S))=1$ holds. Specifically the result $G$ of the extraction of section $L$ from $S$ is computed by

$$
G=\mathcal{T}^{-1}\left(\mathcal{T}(L)^{-1} \cdot \mathcal{T}(S)\right)
$$

The extraction process of $\mathrm{TZ} z_{0}$ by means of elementary sections will lead to a pole-zero simplification of the root $z_{0}$ in each element of the resulting matrix $G$. This simplification has double multiplicity in the case of an entire extraction while it is simple in case of partial extraction. Numerically, this operation is best implemented by performing a polynomial long division of numerators and denominator (of $G$ ) by the factor $\left(s-z_{0}\right)^{k}$ where $k \in\{1,2\}$ : an operation available for example in matlab under the function $\operatorname{deconv}()$.

As for notations, we will denote for example in the case of the two triplet topology,

$$
\left(S^{1}, G\right)=\operatorname{Ext}\left(S,\left\{(\infty, e),\left(z_{1}, e\right),(\infty, p)\right\}\right)
$$

the functional operation that yields the decomposition

$$
S=S^{1} \circ G
$$

via successive extraction of TZs by means of elementary sections. The sequence $\left\{(\infty, e),\left(z_{1}, e\right),(\infty, p)\right\}$ specifies how $S^{1}$ is obtained: it stands for the entire (noted $e$ ) extraction of a $\mathrm{TZ}$ at infinity followed by the entire extraction of a $\mathrm{TZ}$ at $z_{1}$ and a partial (noted $p$ ) extraction of a TZ at infinity.

For partial extraction, the choice of the angular derivative $\zeta_{0}$ of the elementary section is set to $\zeta_{0}=2 \zeta$ in the notations of section II-B1. This rather arbitrary choice, is merely related to the splitting factor $\alpha$ of common resonators and has, as the order selected for the TZ's extraction, no influence on the eventually synthesized circuit obtained by the assembly of the sub-circuits. In (15), $G$ is the two port response that remains after the extraction of $S_{1}$, that is $S=S^{1} \circ G$ : in our simple example the decomposition is complete and we set $S^{2}=G$. A detailed numerical example of the synthesis process will be given after the introduction of coupled resonator models with frequency-dependent couplings.

\section{Dispersively Coupled Resonator Circuits}

\section{A. Dispersive coupling component}

Coupled resonator circuits [1] are usually considered to include only frequency-independent coupling elements that are characterised by a mutual admittance parameter $j M_{k, l}$ between circuit $k$ and $l$. More recently, in [6]-[10], structures able to realize adjustable dispersive coupling elements have been reported in which the mutual admittance is modelled as

$$
j \mathbf{M}_{k, l}=j\left(\mathbf{M o}_{k, l}+\mathbf{M} \mathbf{d}_{k, l} \omega\right)
$$

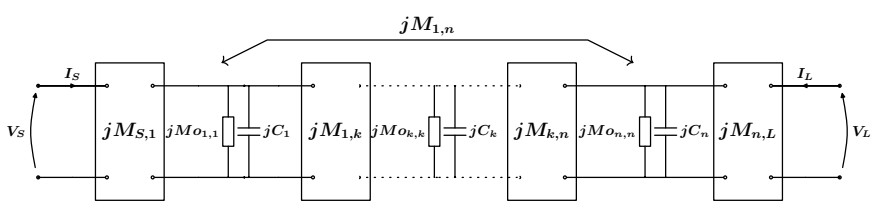

Fig. 5. Low-pass coupled-resonator circuit.

that is linearly varying with frequency. The $2 \times 2$ admittance matrix of such a coupling element exhibits a pole at infinity whose residue, as a consequence of the supposedly lossless nature of the ideal coupling mechanism, needs to be positive semi-definite. If no self-coupling terms are considered this residue is given by,

$$
\mathcal{G}_{k, l}=\left(\begin{array}{cc}
0 & M d_{k, l} \\
M d_{k, l} & 0
\end{array}\right)
$$

and $\operatorname{det}\left(\mathcal{G}_{k, l}\right)=-\left(M d_{k, l}\right)^{2}<0$ indicates that the passivity hypothesis is violated. This calls for the presence of self admittance parameters in the description of the coupling element that we will for simplicity fix to $j\left|\mathbf{M d}_{k, l}\right|$ at both ports of the coupling structure (this choice ensures the residue condition at $\infty$ ). The relations between currents and tensions at the port of such a coupling element (see Fig. 4) are given by:

$$
\left\{\begin{aligned}
I_{k} & =j\left|\mathbf{M d}_{k, l}\right| \omega V_{k}+j\left(\mathbf{M} \mathbf{d}_{k, l} \omega+\mathbf{M} \mathbf{o}_{k, l}\right) V_{l} \\
I_{l} & =j\left(\mathbf{M d}_{k, l} \omega+\mathbf{M} \mathbf{o}_{k, l}\right) V_{k}+j\left|\mathbf{M} \mathbf{d}_{k, l}\right| \omega V_{l}
\end{aligned}\right.
$$

\section{B. State space form}

We now consider a coupled-resonator circuit, see Fig. 5, where all couplings between circuits might be dispersive and defined as in (16) while the input and output couplings denoted by $M_{S, k}, M_{k, L}$ with $k \in 1 \ldots n$ are considered frequencyinvariant. If $U_{k}$ denotes the voltage in the $k^{\text {th }}$ circuit, Kirchhoff's law at the $k^{\text {th }}$ resonator circuit express as:

$$
\begin{aligned}
& j \omega\left(C_{k}+\sum_{i \in\{1 \ldots n\} \neq k}\left|\mathbf{M} \mathbf{d}_{i, k}\right|\right) U_{k}+\sum_{i \in\{1 \ldots n\}} j \mathbf{M} \mathbf{o}_{k, i} U_{i} \\
& +j \mathbf{M} \mathbf{o}_{S, k} V_{s}+j \mathbf{M} \mathbf{o}_{k, L} V_{L} \\
& =0
\end{aligned}
$$

Passing to the time domain and taking as state vector $x=j U$ leads to following normalized state space form [31] of the underlying dynamical system associated to the admittance of the circuit,

$$
\left\{\begin{array}{l}
(\mathbf{M d}) \dot{x}=-j \mathbf{M o} x+\mathbf{B} V \\
I=\mathbf{B}^{t} x
\end{array}\right.
$$

where $V$ and $I$ are $2 \times 1$ vectors containing the input voltages and output currents at the source and load ports. The matrix $\mathbf{B}$ is $n \times 2$ and contains the source and load to resonator couplings, that is $\mathbf{B}_{k, 1}=\mathbf{M} \mathbf{o}_{S, k}$ and $\mathbf{B}_{k, 2}=\mathbf{M} \mathbf{o}_{k, L}$ for $k \in\{1 \ldots n\}$. By a slight abuse of notation $\mathbf{M d}$ and $\mathbf{M o}$ represent here $n \times n$ matrices: the elements of Mo are the 
couplings $\mathbf{M o}_{k, l}$, while off diagonal terms of $\mathbf{M d}$ are the dispersive slops $\mathbf{M d}_{k, l}$ as defined in (16). As for the diagonal terms of the matrix Md we define, according to (17):

$$
\mathbf{M d}_{k, k}=C_{k}+\sum_{i \in\{1 \ldots n\} \neq k}\left|\mathbf{M d}_{i, k}\right|
$$

This latter equation illustrates the loading mechanism of the resonators by dispersive couplings. From the definition of $\mathbf{M d}$ we conclude that it can be written as the sum of a semidefinite matrix built on the residues at infinity of each of the dispersive coupling elements and a positive definite diagonal matrix containing the $C_{i}^{\prime} s>0$. Hence $\mathbf{M d}$ is positive definite, and in particular invertible. This property was postulated in [11] where dispersive couplings were first considered in lowpass equivalent circuits: we have shown here that it is a natural consequence of the passivity of each individual dispersive coupling mechanism.

Proposition 3.1: We call a triplet $(\mathbf{M d}, \mathbf{M o}, \mathbf{B})$ a circuital realization associated to the low-pass circuit of Fig. 5, for which we give elementary properties.

i) Admittance formula: Let $(\mathbf{M d}, \mathbf{M o}, \mathrm{B})$ be a circuital realization, $\mathbf{M d}, \mathbf{M o}, \mathrm{B}$ all real and $\mathbf{M d}$ positive definite, then its admittance is given by

$$
Y=\mathbf{B}(s \mathbf{M d}+j \mathbf{M o})^{-1} \mathbf{B}^{t}=-j \mathbf{B}(\omega \mathbf{M d}+\mathbf{M o})^{-1} \mathbf{B}^{t}
$$

is a $2 \times 2$ strictly proper (i.e. 0 at infinity), reciprocal, lossless positive real transfer function.

ii) Dispersion-less realization: If $Y$ is a $2 \times 2$ strictly proper, reciprocal, lossless positive real transfer function there exists a circuital realization $(\mathbf{M d}, \mathbf{M o}, \mathbf{B})$ to it, where $\mathbf{M d}=I d$ and $(\mathbf{M o}, \mathbf{B})$ are real.

iii) Congruent transformations: Suppose $(\mathbf{M d}, \mathbf{M o}, \mathbf{B})$ and $\left(\mathbf{M d}^{\prime}, \mathbf{M o} \mathbf{o}^{\prime}, \mathbf{B}^{\prime}\right)$ are two minimal circuital realizations of McMillan degree $n$ with the same admittance matrix $Y$, then there exists a non-singular $n \times n$ matrix $P$ such that,

$$
\mathbf{M d}^{\prime}=P^{t} \mathbf{M d} P, \mathbf{M o} \mathbf{o}^{\prime}=P^{t} \mathbf{M o} P, \mathbf{B}^{\prime}=P^{t} \mathbf{B}
$$

Conversely, any realization $\left(\mathbf{M d}^{\prime}, \mathbf{M o} \mathbf{o}^{\prime}, \mathbf{B}^{\prime}\right)$ defined as above for an invertible $P$ has the same admittance matrix as (Md, Mo, B).

iv) Shortest path rule: We consider the usual coupling graph associated to a coupling topology and defined as described: every resonator is represented by a node, and two additional separate nodes are drawn to symbolize the input and output. Edges of length one are drawn between the input (resp. output) node and a resonator $k$ if the corresponding $\mathbf{M o}_{S, k}$ (resp. $\mathbf{M o}_{k, L}$ ) coupling element is present in the circuit. Edges of length one are drawn between resonators nodes $k$ and $l$ if a frequency-invariant coupling $\mathbf{M} \mathbf{o}_{k, l}$ (and $\mathbf{M} \mathbf{d}_{k, l}=0$ ) is nonzero. Eventually, an edge of length zero is drawn between resonator $k$ and $l$ if a frequency dependent couplings $\mathbf{M} \mathbf{d}_{k, l}$ is present in the circuit. Consider a circuit with $n$ resonators. Let $c$ be the length of the shortest path in the coupling graph between input and output, then the scattering matrix of this circuit can maximally possess $(\mathbf{n}+\mathbf{1}-\mathbf{c})$ TZs at finite frequency.

A sketch of the proof is given in the appendix in section VI.

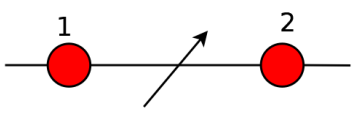

Fig. 6. Topology of dispersive duplet.

\section{Direct Computation of congruent transformations}

Equation (20) might shed some light on circuit synthesis problems associated with circuits with dispersive couplings. As opposed to the classical non-dispersive setting we are in presence of three parts of the coupling matrix $(\mathbf{M d}, \mathbf{M o}, \mathbf{B})$ whose topology need to be controlled by means of congruent transformations (general invertible matrices applied as in (20)). These transformations are the exact extensions of the similarity transforms used for circuit reconfiguration purposes in the classical non-dispersive case. This aspect is of course hidden in the sequence of scaling and orthogonality transformations that were introduced to tackle the associated synthesis problems [19]-[21] that can be seen as a rather indirect, and sometimes tedious, way to compute relevant congruent transformations: we present here a procedure for direct computation of congruent transformations adapted to canonical dispersive structures.

For all topologies hereafter we start from a circuital realization $\mathcal{R}=(\mathbf{M d}=I d, \mathbf{M o}, \mathbf{B})$ of the response $Y$ to be synthesized. This can be for example given by the canonical transversal form of $Y$ which is readily obtained from its partial fraction expansion [1], [4], [31]. We will denote by $\left(w_{1}, w_{2}\right)=\mathbf{B}$ the two $n \times 1$ column vectors constituting $\mathbf{B}$. If $u, v$ are two $n \times 1$ column vectors, $\langle u, v\rangle=u^{t} v$ will denote the classical dot product. Our synthesis problems will consist in the determination of a matrix $P$ such that the realization

$$
\mathcal{R}^{\prime}=\left(\mathbf{M d}^{\prime}=P^{t} P, \mathbf{M o}^{\prime}=P^{t} \mathbf{M o} P, \mathbf{B}^{\prime}=P^{t} \mathbf{B}\right)
$$

is compatible with the targeted coupling topology. We denote by $\left(v_{1}, v_{2}, \ldots v_{n}\right)=P$ the column vectors of the matrix $P$ to be determined. In terms of dot product between vectors (20), recalled in $\mathcal{R}^{\prime}$, translates into,

$$
\begin{cases}\left(\mathbf{M d}^{\prime}\right)_{k, l} & =<v_{k}, v_{l}> \\ \left(\mathbf{M o}^{\prime}\right)_{k, l} & =<v_{k}, \mathbf{M o} v_{l}> \\ \left(\mathbf{B}^{\prime}\right)_{k, l} & =<w_{k}, v_{l}>\end{cases}
$$

In that light the synthesis problem amounts to determine a set of basis vectors $v_{k}$ that satisfy certain orthogonality relations imposed by the targeted coupling topology. Such an approach was for example used in [4] to prove uniqueness of the canonical arrow form.

1) Dispersive Duplet (see Fig. 6): As a consequence of the shortest path rule this form accommodates a response of degree 2 with $1 \mathrm{TZ}$. Two vectors defining the congruent transformation need to be determined. The target matrix $\mathbf{B}^{\prime}$ verifies $\mathbf{B}^{\prime}(1,2)=0$ and $\mathbf{B}^{\prime}(2,1)=0$ according to (21) translates into $\left\langle v_{1}, w_{2}\right\rangle=0$ and $\left\langle v_{2}, w_{1}\right\rangle=0$. The directions of $v_{1}$ and $v_{2}$ are therefore uniquely determined by

$$
v_{1}=w_{1}-\frac{<w_{1}, w_{2}>}{<w_{2}, w_{2}>} w_{2}, v_{2}=w_{2}-\frac{\left.<w_{2}, w_{1}\right\rangle}{\left.<w_{1}, w_{1}\right\rangle} w_{1}
$$

A rescaling operation by means of a diagonal congruent transformation can eventually be applied in order to obtain 


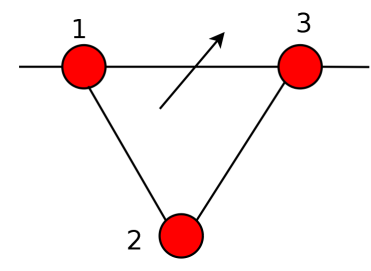

Fig. 7. Topology of dispersive triplet.

a dispersive matrix $\mathbf{M d}^{\prime}$ with unitary diagonal entries. Note that this simple orthogonalization technique combined with our decomposition technique in the frequency domain yields an alternative synthesis procedure to the $n-1$ inline cascaded dispersive duplets form presented in [21] and obtained there via rather complicate sequences of scaling and similarity transforms.

Note that we made the implicit generic hypothesis that $\operatorname{rank}\left(\left\{w_{1}, w_{2}\right\}\right)=2$. If this fails, the matrix $P$ becomes singular. This might happen for very special response classes, for example those where $Y_{1,1}=Y_{2,2}$ holds and where the $\mathrm{TZ}$ lies "in between" two resonating frequencies on the $j \omega$ axis (in this case $w_{1}=w_{2}$ for the transversal form): for this singular class of responses, there does not exist a dispersive duplet form. We will leave to further work the careful study of such singular and non-generic classes of responses and note that similar exceptional behaviours were already observed in [32].

2) Dispersive Triplet (see Fig. 7): This building block is compatible with order 3 responses with at most 2 TZs, as the minimal source to load path is here of length 2. Couplings $(1,2)$ and $(2,3)$ are non-dispersive and therefore $v_{1}$ and $v_{3}$ are orthogonal to $v_{2}$, but the shape of $\mathbf{B}^{\prime}$ indicates that $w_{1}$ and $w_{2}$ are orthogonal to $v_{2}$, which shows (always under the same generic rank hypothesis) that $\left\{v_{2}\right\}^{\perp}=\operatorname{span}\left(w_{1}, w_{2}\right)$. Consequently, as in the duplet case we have

$$
v_{1}=w_{1}-\frac{<w_{1}, w_{2}>}{<w_{2}, w_{2}>} w_{2}, v_{3}=w_{2}-\frac{<w_{2}, w_{1}>}{<w_{1}, w_{1}>} w_{1}
$$

Eventually the direction of $v_{2}$ is uniquely determined, and given by

$$
v_{2}=v_{1} \times v_{3}
$$

where $\times$ is the usual cross product in $\mathbb{R}^{3}$. Under the generic hypothesis that $\boldsymbol{M o} v_{1}$ does not belong to $\operatorname{span}\left(v_{1}, v_{2}\right)=$ $\operatorname{span}\left(w_{1}, w_{2}\right)$ and observing that $\left\{v_{1}, w_{2}\right\}$ is an orthogonal basis of $\operatorname{span}\left(w_{1}, w_{2}\right)$, we have the following alternative expression for the direction of $v_{2}$ :

$$
v_{2}=\mathbf{M o} v_{1}-\frac{<\mathbf{M o} v_{1}, v_{1}>}{<v_{1}, v_{1}>} v_{1}-\frac{<\mathbf{M o} v_{1}, w_{2}>}{<w_{2}, w_{2}>} w_{2}
$$

3) Dispersive Quadruplet with Two or Three TZs ( see Fig. 8): This topology is compatible with order 4 responses with up to 3 TZs. The shortest path rule shows that if only 2 TZs are to be realised then the coupling $(1,4)$ becomes nondispersive. A similar reasoning as in the triplet case shows that $\operatorname{span}\left(v_{1}, v_{4}\right)=\operatorname{span}\left(w_{1}, w_{2}\right)$ and therefore,

$$
v_{1}=w_{1}-\frac{<w_{1}, w_{2}>}{<w_{2}, w_{2}>} w_{2}, v_{4}=w_{2}-\frac{<w_{2}, w_{1}>}{<w_{1}, w_{1}>} w_{1}
$$

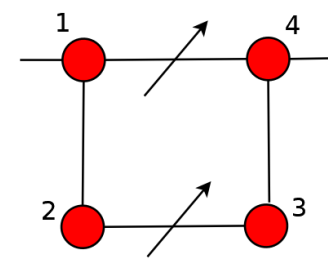

Fig. 8. Topology of dispersive quadruplet.

From the non-dispersive nature of coupling $(1,2)$ and the absence of couplings $(2,4)$, we conclude that the direction of $v_{2}$ is entirely characterised by its orthogonality to the three vectors $v_{1}, v_{4}$ and $\mathbf{M o} v_{4}$. At this point we will suppose that $\left\{v_{1}, v_{4}, \mathbf{M o} v_{1}, \mathbf{M o} v_{4}\right\}$ is a linearly independent family of vectors: this property is again generically true for almost all but a small set of singular responses. In order to determine the direction orthogonal to $\operatorname{span}\left(v_{1}, v_{4}, \mathbf{M o} v_{4}\right)=$ $\operatorname{span}\left(v_{1}, w_{2}, \mathbf{M o} v_{4}\right)$ we first determine an orthogonal basis of this vector space. The vectors $v_{1}, w_{2}$ are already orthogonal, we therefore only need to introduce $v_{t 1}$ defined as

$$
v_{t 1}=\operatorname{Mo} v_{4}-\frac{<\operatorname{Mo} v_{4}, v_{1}>}{<v_{1}, v_{1}>} v_{1}-\frac{<\operatorname{Mo} v_{4}, w_{2}>}{<w_{2}, w_{2}>} w_{2}
$$

and $\left\{v_{1}, w_{2}, v_{t 1}\right\}$ yields the desired basis. The direction of vector $v_{2}$ is then defined by

$$
\begin{aligned}
v_{2}=\operatorname{Mo} v_{1}-\frac{<\operatorname{Mo} v_{1}, v_{1}>}{<v_{1}, v_{1}>} v_{1}- & \frac{<\operatorname{Mo} v_{1}, w_{2}>}{<w_{2}, w_{2}>} w_{2} \\
- & \frac{<\mathbf{M o} v_{1}, v_{t 1}>}{<v_{t 1}, v_{t 1}>} v_{t 1}
\end{aligned}
$$

Symmetrically, we define

$$
v_{t 2}=\mathbf{M o} v_{1}-\frac{<\mathbf{M o} v_{1}, v_{1}>}{<v_{1}, v_{1}>} v_{1}-\frac{<\mathbf{M o} v_{1}, w_{2}>}{<w_{2}, w_{2}>} w_{2}
$$

and obtain $v_{3}$ as,

$$
\begin{aligned}
v_{3}=\operatorname{Mo} v_{4}-\frac{<\operatorname{Mo} v_{4}, v_{1}>}{<v_{1}, v_{1}>} v_{1}- & \frac{<\operatorname{Mo} v_{4}, w_{2}>}{<w_{2}, w_{2}>} w_{2} \\
- & \frac{<\mathbf{M o} v_{4}, v_{t 2}>}{<v_{t 2}, v_{t 2}>} v_{t 2}
\end{aligned}
$$

This Gramm-Schmidt type orthogonalization procedure can be extended to higher degree sections in order to synthesize the general dispersive folded form presented in [19] and obtained via scaling and similarity transforms applied to an initial lattice form. Our vector based approach shows that, up to the usual sign changes, this dispersive folded canonical form is generically unique.

\section{Synthesis EXAMPLES}

In this section, a 6-pole filter with $4 \mathrm{TZs}$ and a 10-pole filter with 8 TZs in mixed topologies will be used to demonstrate and validate the proposed synthesis technique. The detailed process of the synthesis will mainly be given in the first example, including the extraction of elementary scattering sections yielding the frequency domain decomposition as well as the synthesis of the coupling matrices of each sub-filter. The overall coupling matrix can be eventually obtained by 


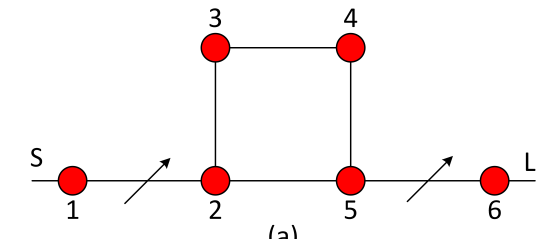

(a)

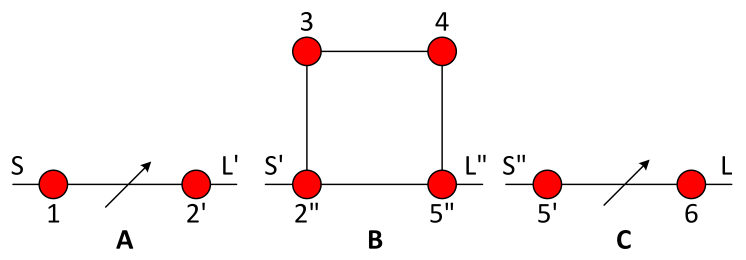

(b)

Fig. 9. (a) Topology of a six-pole filter with a quadruplet and 2 dispersive couplings. (b) Seperating the filter in (a) into 3 sub-filters labeled A, B, and C.

cascading the resultant sub-circuits. Finally, a physical implementation of dispersive couplings within coaxial resonators will be proposed and result in a practical validation of the method. The second example deals with a more complex topology that cascades all of the 4 basic blocks introduced in III-C. It is presented to demonstrate the versatility and numerical accuracy, even for higher orders, of the proposed synthesis method.

\section{A. Synthesis and Design of a Six-Pole Dispersive Filter}

This filter is centered at $f_{0}=2.59 \mathrm{GHz}$ with the bandwidth $B W=200 \mathrm{MHz}$. The topology of it is depicted in Fig. 9(a), with a symmetric quadruplet and two dispersive couplings. As shown in Fig. 9(b), this filter can be separated into three blocks, which are two dispersive duplets $(1-2)$ (sub-filter A), (5-6) (sub-filter C), and a symmetric quadruplet $(2-3-4-5)$ (sub-filter B). We first synthesize a degree 6 response with 4 symmetric TZs, two at $\pm 1.5 j$ assigned to the quadruplet and two at $\pm 3 j$ assigned to the two dispersive duplets, respectively. The desired return loss level is $23 d B$. The Belevitch form of the response is defined as

$$
S=\frac{1}{E(s)}\left[\begin{array}{ll}
F(s) & P(s) \\
P(s) & H(s)
\end{array}\right]
$$

where $E$ is a unitary polynomial computed from $F$ and $P$ by the Feldtqueller equation and $P=-e^{i \theta} P^{*}$ as well as $H=e^{i \theta} F^{*}$ hold for a particular phase factor $e^{i \theta}$. The coefficients of the characteristic polynomials of our target quasi-elliptic response scattering matrix $S$ are listed in Table I. For all subsequent responses we will keep this $F, P, H, E$ denomination for their Belevitch forms. Note that in the Table the coefficients are by descending degree order, the constant term coming last.

The extraction process is conducted within the following steps:

1) Extraction of sub-filter A: Suppose that there is a finite $\mathrm{TZ}$ at $+3 j$ in sub-filter $\mathrm{A}$ apart from the $\mathrm{TZ}$ at infinity. Its
TABLE I

Coefficients of Characteristic Polynomials of the Remaining SCATTERING MATRICES FOR DIFFERENT STAGES REPRESENTED BY DIFFERENT SUPERSCRIPTS

\begin{tabular}{|c|c|c|c|c|c|c|}
\hline$F$ & $F^{A 1}$ & $F^{A}$ & $F^{B 1}$ & $F^{B 2}$ & $F^{B 3}$ & $F^{B}$ \\
\hline 1 & 1 & 1 & $\begin{array}{l}0.734+ \\
0.680 j\end{array}$ & $\begin{array}{l}0.734+ \\
0.680 j\end{array}$ & $\begin{array}{l}0.734+ \\
0.680 j\end{array}$ & 1 \\
\hline 0 & $\begin{array}{l}1.012+ \\
0.519 j\end{array}$ & $\begin{array}{l}0.912+ \\
0.776 j\end{array}$ & $\begin{array}{l}1.158- \\
0.376 j\end{array}$ & $\begin{array}{l}0.401+ \\
1.023 j\end{array}$ & $\begin{array}{l}0.668+ \\
0.873 j\end{array}$ & $\begin{array}{l}1.154- \\
0.147 j\end{array}$ \\
\hline 1.588 & $\begin{array}{l}1.833+ \\
0.551 j\end{array}$ & $\begin{array}{l}2.046+ \\
0.811 j\end{array}$ & $\begin{array}{l}1.878- \\
0.030 j\end{array}$ & $\begin{array}{l}0.490+ \\
1.034 j\end{array}$ & $\begin{array}{c}0.418+ \\
0.659 j\end{array}$ & $\begin{array}{c}1.510+ \\
0.399 j\end{array}$ \\
\hline 0 & $\begin{array}{l}1.133+ \\
0.820 j\end{array}$ & $\begin{array}{l}1.3290+ \\
1.299 j\end{array}$ & $\begin{array}{l}1.430- \\
0.707 j\end{array}$ & $\begin{array}{c}0.026+ \\
0.526 j\end{array}$ & & \\
\hline 0.653 & $\begin{array}{l}0.693+ \\
0.460 j\end{array}$ & $\begin{array}{l}1.019+ \\
0.756 j\end{array}$ & $\begin{array}{c}0.826- \\
0.282 j\end{array}$ & & & \\
\hline 0 & $\begin{array}{c}0.156+ \\
0.184 j\end{array}$ & $\begin{array}{c}0.312+ \\
0.368 j\end{array}$ & & & & \\
\hline 0.043 & & & & & & \\
\hline
\end{tabular}

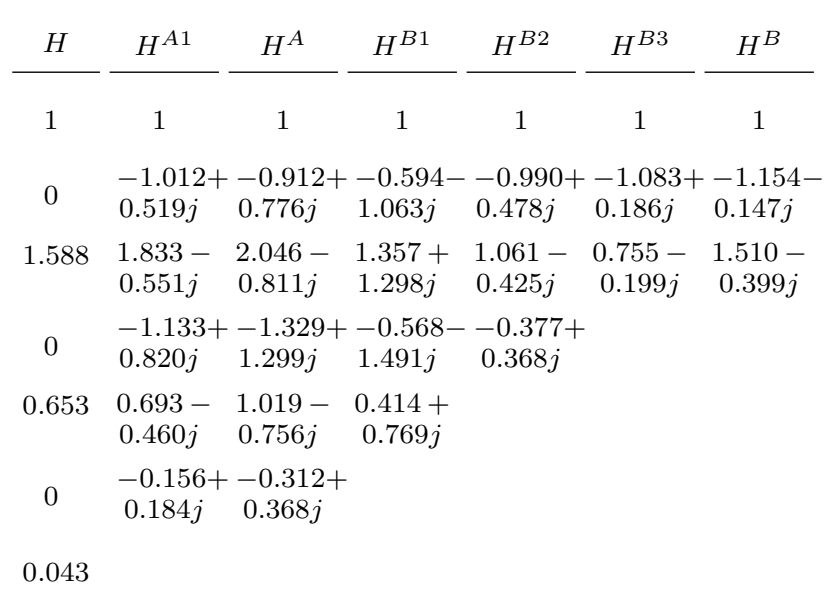

\begin{tabular}{|c|c|c|c|c|c|c|}
\hline$E$ & $E^{A 1}$ & $E^{A}$ & $E^{B 1}$ & $E^{B 2}$ & $E^{B 3}$ & $E^{B}$ \\
\hline 1 & 1 & 1 & 1 & 1 & 1 & 1 \\
\hline 2.226 & $\begin{array}{l}1.214+ \\
0.519 j\end{array}$ & $\begin{array}{l}1.315+ \\
0.776 j\end{array}$ & $\begin{array}{l}1.633- \\
1.063 j\end{array}$ & $\begin{array}{l}1.237+ \\
0.478 j\end{array}$ & $\begin{array}{l}1.143+ \\
0.186 j\end{array}$ & $\begin{array}{l}1.274- \\
0.147 j\end{array}$ \\
\hline 4.066 & $\begin{array}{l}2.057+ \\
0.604 j\end{array}$ & $\begin{array}{l}2.495+ \\
0.917 j\end{array}$ & $\begin{array}{l}2.507- \\
1.053 j\end{array}$ & $\begin{array}{l}1.331+ \\
0.653 j\end{array}$ & $\begin{array}{c}0.810+ \\
0.230 j\end{array}$ & $\begin{array}{c}1.631+ \\
0.461 j\end{array}$ \\
\hline 4.554 & $\begin{array}{l}1.459+ \\
0.879 j\end{array}$ & $\begin{array}{l}1.982+ \\
1.417 j\end{array}$ & $\begin{array}{l}1.911- \\
1.253 j\end{array}$ & $\begin{array}{c}0.440+ \\
0.572 j\end{array}$ & & \\
\hline 3.787 & $\begin{array}{l}0.882+ \\
0.528 j\end{array}$ & $\begin{array}{l}1.396+ \\
0.893 j\end{array}$ & $\begin{array}{c}1.035- \\
0.489 j\end{array}$ & & & \\
\hline 2.044 & $\begin{array}{c}0.230+ \\
0.217 j\end{array}$ & $\begin{array}{c}0.459+ \\
0.433 j\end{array}$ & & & & \\
\hline 0.614 & & & & & & \\
\hline
\end{tabular}

\begin{tabular}{|c|c|c|c|c|c|c|}
\hline$P$ & $P^{A 1}$ & $P^{A}$ & $P^{B 1}$ & $P^{B 2}$ & $P^{B 3}$ & $P^{B}$ \\
\hline $0.030 j$ & $0.030 j$ & $-0.061 j$ & $\begin{array}{l}0.102+ \\
0.040 j\end{array}$ & $\begin{array}{l}0.102+ \\
0.040 j\end{array}$ & $\begin{array}{l}0.102+ \\
0.040 j\end{array}$ & -0.219 \\
\hline 0 & -0.091 & 0.182 & $\begin{array}{l}-0.120+ \\
0.306 j\end{array}$ & $\begin{array}{l}-0.180+ \\
0.459 j\end{array}$ & $\begin{array}{l}-0.120+ \\
0.306 j\end{array}$ & $-0.658 j$ \\
\hline $0.340 j$ & $0.0680 j$ & $-0.136 j$ & $\begin{array}{l}0.230+ \\
0.090 j\end{array}$ & $\begin{array}{l}-0.459- \\
0.180 j\end{array}$ & & \\
\hline 0 & -0.204 & 0.408 & $\begin{array}{c}-0.270+ \\
0.689 j\end{array}$ & & & \\
\hline $0.613 j$ & & & & & & \\
\hline
\end{tabular}


scattering matrix $S^{A}$ can be obtained by extracting these two TZs from $S$ as

$$
\left(S^{A}, G^{A}\right)=\operatorname{Ext}(S,\{(+3 j, e),(\infty, p)\})
$$

where $G^{A}$ is the remaining scattering matrix after the extraction of $S^{A}$.

To find $S^{A}$, we first entirely extract the finite $\mathrm{TZ}$ at $+3 j$ as

$$
\left(S^{A 1}, G^{A 1}\right)=\operatorname{Ext}(S,(+3 j, e))
$$

$S^{A 1}$ is established by (14) with $\gamma=S_{1,1}(3 j)=$ $0.715+0.700 j$ and $\zeta_{0}=\operatorname{Ang}\left(S_{1,1}\right)[3 j]=-0.282$. The coefficients of the characteristic polynomials $F^{A 1}(s), H^{A 1}(s), E^{A 1}(s)$, and $P^{A 1}(s)$, of the remaining scattering matrix $G^{A 1}$ are listed in Table I. Note that all of the listed coefficients are corresponding to the characteristic polynomials of the remaining scattering matrix $G$ with the corresponding superscript except that the initial one corresponds to $S$.

We can observe that the degree of the remaining response is reduced by 1 compared to the original scattering matrix due to the entire extraction of the TZ, which is

$$
\operatorname{deg}\left(G^{A 1}\right)=\operatorname{deg}(S)-1
$$

Then, a TZ at infinity is partially extracted to obtain the other pole of the sub-filter and with the order of the remaining polynomials staying unchanged as

$$
\left(S^{A 2}, G^{A}\right)=\operatorname{Ext}\left(G^{A 1},(\infty, p)\right)
$$

Note that if we finish this extraction, the scattering matrix $S^{A}$ of sub-filter A can be found as

$$
S^{A}=S^{A 1} \circ S^{A 2}
$$

Thus, the remaining response directly becomes $G^{A}$, the same as what is in (22).

In this case, $S^{A 2}$ is established by (13) with $\gamma=1$ and we choose $\zeta_{0}=2 \zeta=-0.403$ to do the partial extraction. As shown in Table I, the degree of $G^{A}$ is the same as $G^{A 1}$, showing that the TZ at infinity is partially extracted, which corresponds to the extraction of resonator $2^{\prime}$ in Fig. 9 (b).

Chaining the two extracted elementary sections together by (23), we find the scattering matrix of sub-filter A. The nondispersive coupling matrix can be formulated and then further transformed to the dispersive coupling matrix in the desired topology using the computation in Section III-C1 as

$$
\begin{gathered}
\mathbf{M o}^{A}=\left[\begin{array}{cc}
-0.519 & 1.222 \\
1.222 & -0.909
\end{array}\right] \quad \mathbf{B}^{A}=\left[\begin{array}{cc}
1.006 & 0 \\
0 & 0.410
\end{array}\right] \\
\mathbf{M d}^{A}=\left[\begin{array}{cc}
1 & -0.407 \\
-0.407 & 1
\end{array}\right]
\end{gathered}
$$

2) Extraction of sub-filter B: Sub-filter $B$ is a regular quadruplet with two symmetric TZs at $\pm 1.5 j$ and two infinite TZs. The scattering matrix $S^{B}$ of this sub-filter can be obtained by extracting all these TZs from the current remaining response $G^{A}$ as

$\left(S^{B}, G^{B}\right)=\operatorname{Ext}\left(G^{A},\{(\infty, e),(+1.5 j, e),(-1.5 j, e),(\infty, p)\}\right)$ where $G^{B}$ is the remaining scattering matrix after the extraction of $S^{B}$. Note that the last resonator is shared with sub-filter $\mathrm{C}$, so one of the infinite TZs should be partially extracted.

Similar to the procedure to extract sub-filter A, we first entirely extract a $\mathrm{TZ}$ at infinity as

$$
\left(S^{B 1}, G^{B 1}\right)=\operatorname{Ext}\left(G^{A},(\infty, e)\right)
$$

where $S^{B 1}$ is given by (13) with $\gamma=1$ and $\zeta_{0}=\zeta=-0.403$. The coefficients of the characteristic polynomials of the remaining response $G^{B 1}$ are listed in Table I. An order reduction of 1 is observed for all characteristic polynomials compared to those of $G^{A}$, which confirms that the TZ is fully extracted.

Next, the finite $\mathrm{TZ}$ at $+1.5 j$ is entirely extracted as

$$
\left(S^{B 2}, G^{B 2}\right)=\operatorname{Ext}\left(G^{B 1},(+1.5 j, e)\right)
$$

with $\gamma=-0.581-0.814 j$ and $\zeta_{0}=\zeta=-4.999$ to construct the elementary section $S^{B 2}$. The degree of the remaining scattering matrix $G^{B 2}$ is reduced by 1 compared to $G^{B 1}$ as shown in Table I.

Then, we entirely extract the other finite $\mathrm{TZ}$ at $-1.5 j$ as

$$
\left(S^{B 3}, G^{B 3}\right)=\operatorname{Ext}\left(G^{B 2},(-1.5 j, e)\right)
$$

with $S^{B 3}$ constructed with $\gamma=0.830+0.558 j$ and $\zeta_{0}=\zeta=$ -0.128 . The degree of $G^{B 3}$ is reduced by 1 again.

Finally, the last $\mathrm{TZ}$ at infinity is partially extracted as

$$
\left(S^{B 4}, G^{B}\right)=\operatorname{Ext}\left(G^{B 3},(\infty, p)\right)
$$

with $\gamma=0.734+0.680 j$ and $\zeta_{0}=2 \zeta=-0.119$ for $S^{B 4}$. The scattering matrices of all the 4 elementary sections are all obtained, the chaining of which is the scattering matrix $S^{B}$ of sub-filter B

$$
S^{B}=S^{B 1} \circ S^{B 2} \circ S^{B 3} \circ S^{B 4}
$$

The remaining scattering matrix $G^{B}$ is automatically the scattering matrix $S^{C}$ of sub-filter C.

The coupling matrix corresponding to $S^{B}$ is

$$
\begin{aligned}
\mathbf{M o}^{B} & =\left[\begin{array}{cccc}
0.514 & 0.860 & 0 & -0.300 \\
0.860 & 0.044 & 0.709 & 0 \\
0 & 0.709 & -0.044 & 0.860 \\
-0.300 & 0 & 0.860 & 0.152
\end{array}\right] \\
\mathbf{B}^{B} & =\left[\begin{array}{cccc}
0.449 & 0 & 0 & 0 \\
0 & 0 & 0 & 0.244
\end{array}\right]^{t}, \mathbf{M d}^{B}=I d
\end{aligned}
$$

which is a classical non-dispersive quadruplet.

3) The remaining sub-filter $C$ : As mentioned in the last step, after extracting sub-filter $A$ and $B$, the remaining section automatically becomes $S^{C}$. The dispersive coupling matrix of sub-filter $\mathrm{C}$ is found to be

$$
\begin{gathered}
\mathbf{M o}^{C}=\left[\begin{array}{ll}
0.354 & 1.222 \\
1.222 & 0.519
\end{array}\right] \quad \mathbf{B}^{C}=\left[\begin{array}{cc}
0.223 & 0 \\
0 & 1.006
\end{array}\right] \\
\mathbf{M d}^{C}=\left[\begin{array}{cc}
1 & 0.407 \\
0.407 & 1
\end{array}\right]
\end{gathered}
$$

Till now, all of the scattering parameters of the sub-filters are extracted and further used to construct the corresponding 
coupling matrices. Then, by means of a diagonal congruent transformation we re-scale the input inverter of the second circuit B such that $\mathbf{M}^{B}\left(S^{\prime}, 2^{\prime \prime}\right)=-\mathbf{M}^{A}\left(2^{\prime}, L^{\prime}\right)$, and its output inverter such that $\mathbf{M}^{B}\left(5^{\prime \prime}, L^{\prime \prime}\right)=-\mathbf{M}^{C}\left(S^{\prime \prime}, 5^{\prime}\right)$. The resultant matrices $\mathbf{M}^{B}, \mathbf{B}^{B}, \mathbf{M} \mathbf{d}^{B}$ are

$$
\begin{gathered}
\mathbf{M o}^{B}=\left[\begin{array}{cccc}
0.429 & -0.786 & 0 & -0.251 \\
-0.786 & 0.044 & 0.709 & 0 \\
0 & 0.709 & -0.044 & -0.786 \\
-0.251 & 0 & -0.786 & 0.127
\end{array}\right] \\
\mathbf{B}^{B}=\left[\begin{array}{ccccc}
-0.410 & 0 & 0 & 0 \\
0 & 0 & 0 & -0.223
\end{array}\right]^{t} \\
\mathbf{M d}^{B}=\left[\begin{array}{cccc}
0.834 & 0 & 0 & 0 \\
0 & 1 & 0 & 0 \\
0 & 0 & 1 & 0 \\
0 & 0 & 0 & 0.834
\end{array}\right]
\end{gathered}
$$

Now, circuits $\mathrm{A}, \mathrm{B}$ and $\mathrm{C}$ can be cascaded by reversing the operation depicted on Fig. 2 and this yields a low-pass circuit compatible with the topology in Fig. 9(a). By further re-normalizing the diagonal terms $(2,2)$ and $(5,5)$ of the so obtained matrix $\mathbf{M d}$, we get

$$
\begin{gathered}
\mathbf{M o}=\left[\begin{array}{cccccc}
-0.519 & 0.902 & 0 & 0 & 0 & 0 \\
0.902 & -0.262 & 0.580 & 0 & -0.137 & 0 \\
0 & 0.580 & 0.044 & 0.709 & 0 & 0 \\
0 & 0 & 0.709 & -0.044 & 0.580 & 0 \\
0 & -0.137 & 0 & 0.580 & 0.262 & 0.902 \\
0 & 0 & 0 & 0 & 0.902 & 0.519
\end{array}\right] \\
\mathbf{M d}=\left[\begin{array}{ccccccc}
1.006 & 0 & 0 & 0 & 0 & 0 \\
0 & 0 & 0 & 0 & 0 & 1.006
\end{array}\right]^{t} \\
\mathbf{B}=\left[\begin{array}{cccccc}
1 & -0.301 & 0 & 0 & 0 & 0 \\
-0.301 & 1 & 0 & 0 & 0 & 0 \\
0 & 0 & 1 & 0 & 0 & 0 \\
0 & 0 & 0 & 1 & 0 & 0 \\
0 & 0 & 0 & 0 & 1 & 0.301 \\
0 & 0 & 0 & 0 & 0.301 & 1
\end{array}\right]
\end{gathered}
$$

We can further verify by transforming the dispersive coupling matrix back to a dispersion-less coupling matrix $\mathbf{M o}^{\prime}$ in the canonical folded form through a congruent transformation (20) $P$ :

$$
\begin{gathered}
P=\left[\begin{array}{cccccc}
1.049 & 0 & 0 & 0 & 0 & 0 \\
0.315 & 0.977 & -0.203 & 0.040 & 0.049 & 0 \\
0 & 0.207 & 0.968 & -0.139 & 0 & 0 \\
0 & 0 & 0.139 & 0.968 & -0.207 & 0 \\
0 & -0.049 & 0.040 & 0.203 & 0.977 & -0.315 \\
0 & 0 & 0 & 0 & 0 & 1.049
\end{array}\right] \\
\mathbf{M d}^{\prime}=P^{t} \mathbf{M d} P=I d
\end{gathered}
$$

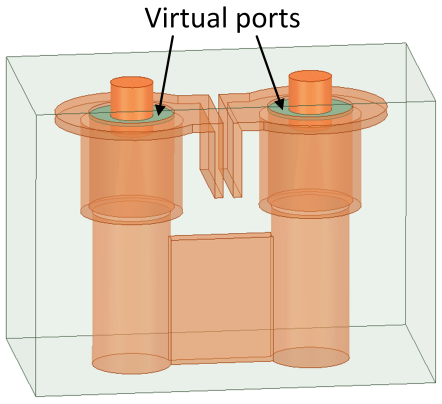

(a)

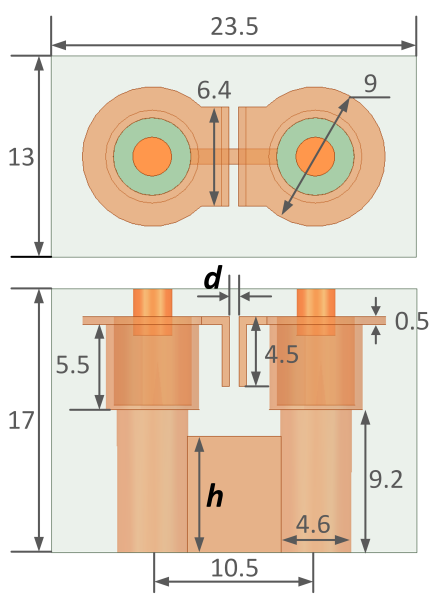

(b)

Fig. 10. (a) EM model of the dispersive coupling structure between two coaxial resonators. (b) Top and front views of the dispersive coupling structure shown in (a).

$$
\begin{aligned}
\mathbf{M o}^{\prime}= & P^{t} \mathbf{M o} P \\
= & {\left[\begin{array}{cccccc}
0 & 0.884 & 0 & 0 & 0 & 0.014 \\
0.884 & 0 & 0.595 & 0 & -0.174 & 0 \\
0 & 0.595 & 0 & 0.726 & 0 & 0 \\
0 & 0 & 0.726 & 0 & 0.595 & 0 \\
0 & -0.174 & 0 & 0.595 & 0 & 0.884 \\
0.014 & 0 & 0 & 0 & 0.884 & 0
\end{array}\right] } \\
& \mathbf{B}^{\prime}=P^{t} \mathbf{B}=\left[\begin{array}{cccccc}
1.055 & 0 & 0 & 0 & 0 & 0 \\
0 & 0 & 0 & 0 & 0 & 1.055
\end{array}\right]^{t}
\end{aligned}
$$

The dispersion-less $\left(\mathbf{M d}^{\prime}, \mathbf{M o} \mathbf{o}^{\prime}, \mathbf{B}^{\prime}\right)$ has exactly the same admittance matrix as the dispersive (Md, Mo, B) by (19). The subsequent design will be conducted following the guidance of the synthesized dispersive coupling matrix $(\mathbf{M d}, \mathbf{M o}, \mathbf{B})$.

The structure depicted in Fig. 10(a) is used to generate a dispersive coupling. This structure is constructed by the two bended edges of the plates on the top of the coaxial resonators, together with a ridge connected between the bottom of the two resonators. Normally, we use only the two parallel bended plates to generate a negative coupling or only the ridge to generate a positive coupling. These couplings are also relatively constant throughout the interested frequency band. However, the combination of these two structures shows a 
dispersive characteristic. This concept is similar to that of the structure in [19] to create a capacitance together with an inductance between two resonators so as to generate a frequency-dependent coupling.

Between each tuning screw and the resonator, there is a virtual port as shown in the figure. We can calculate the coupling value $M$ in the whole frequency band with the Yparameter of this two-port model using the theory in [33] as

$$
M(f)=\frac{2 \Im\left(Y_{12}(f)\right)}{\left.B W \frac{d \Im\left(Y_{11}(f)\right)}{d f}\right|_{f=f_{0}}}
$$

where $\Im(x)$ represents the imaginary part of $x$.

Some basic dimensions are demonstrated on the top view and front view of the structure in Fig. 10(b). The height $h$ of the ridge and the width $d$ of the gap between the two capacitive plates are two critical dimensions that are able to control $M o$ and $M d$ respectively. By simulation with different $h$ and $d$, different coupling coefficients versus frequency are observed in Fig. 11. Generally, the frequency dependence of the coupling coefficients is approximately linear, which is consistent with our hypothesis $M(\omega)=M d \omega+M o$, where $M d$ is the slope and is observed to be negative, $M o$ is the constant part, or the coupling coefficient at the center frequency. The slope parameter can be essentially controlled by the gap value between the plates whereas the core coupling value can be further independently adjusted by the height of the ridge.

In Fig. 11 (a), adjusting the ridge height $h$ can modify $M o$ with $M d$ unchanged. The higher the ridge is, the larger $M o$ becomes. It is observed in Fig. 11(b) that by decreasing the gap width $d$, the absolute value of $M d$ can be increased. However, decreasing $d$ increases the capacitance generated by the two parallel plates, which leads to a decrease in $M o$ simultaneously. Note that this decrease can be compensated by increasing $h$ according to the previous analysis, so that $M d$ and $M o$ can be controlled independently.

The whole filter is designed as shown in Fig. 12 (a), with coupling $(1,2)$ and $(5,6)$ realized by the aforementioned dispersive structure. Note that $\mathbf{M} \mathbf{d}_{5,6}=-\mathbf{M d}_{1,2}$, but $M d$ is regularly negative according to the analysis. Therefore, we change $\mathbf{M o}_{5,6}$ from positive to negative so that $\mathbf{M d}_{5,6}=$ $\mathbf{M d}_{1,2}=-0.283$. This can be realized simply by decreasing the height of the ridge until $\mathbf{M o}_{5,6}=-0.849$. According to the synthesized coupling matrix, the other regular couplings are all positive except for the cross coupling $(2,5)$. This negative coupling is realized by the two parallel plates extended from the top plate edges of resonators 2 and 5. All the sequential positive couplings are realized by ridges and windows.

The photograph of the prototyped filter is shown in Fig. 12 (b). In Fig. 13, we observe that the measured response has a good agreement with that by synthesis, except for a TZ close to the passband in the higher rejection band, which is not exactly symmetric to the other one in the lower rejection band. This is mainly due to the leakage of coupling in the quadruplet. Specifically, there are some parasitic couplings $(2,4)$ and $(3,5)$. Despite this slight mismatch, the good

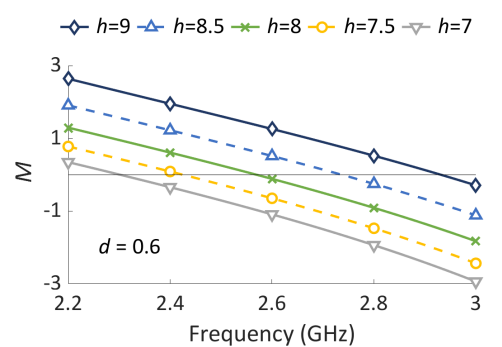

(a)

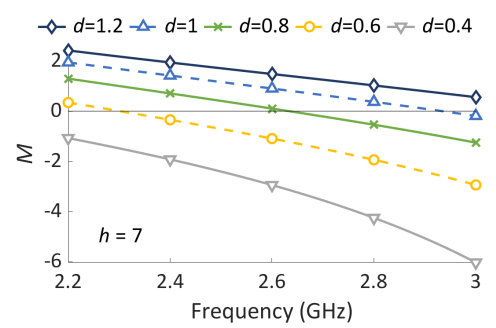

(b)

Fig. 11. Coupling coefficient $M$ versus (a) ridge height $h$ and (b) gap width $d$.

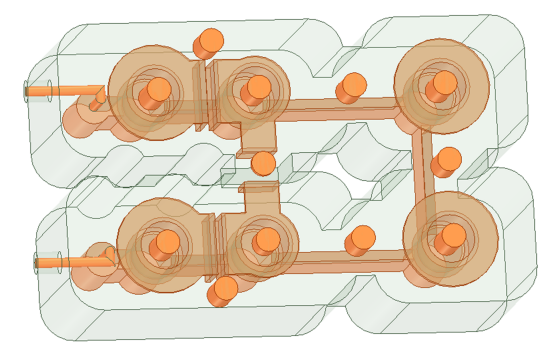

(a)

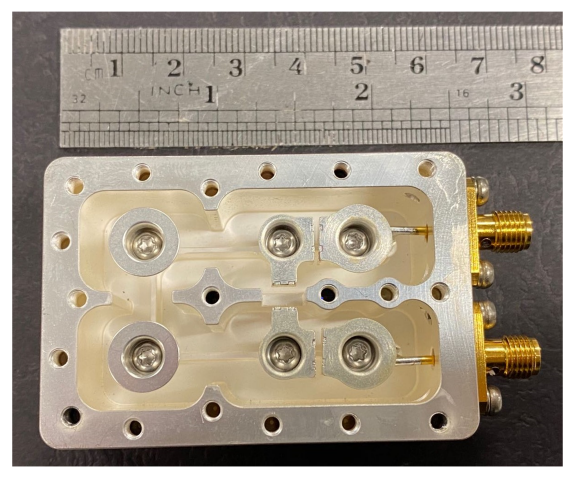

(b)

Fig. 12. (a) EM designed model of the filter with 2 dispersive couplings and a symmetric quadruplet. (b) Photograph of the prototyped filter.

agreement between measurement and synthesis validates the proposed synthesis method.

It is worth noting that the realisation of dispersive couplings is not limited to waveguide and combline filters: implementations have been recently reported in SIW and planar technologies in [34]-[36]. 
TABLE II

Coupling Coefficients of Four Sub-Filters And the Ten-Pole Filter

\begin{tabular}{|c|c|c|c|c|c|c|c|c|c|c|c|c|c|c|c|}
\hline $\mathbf{M o}^{A}$ & $\begin{array}{c}(1,1) \\
-0.440 \\
\end{array}$ & $\begin{array}{l}(1,2) \\
1.126 \\
\end{array}$ & $\begin{array}{c}(2,2) \\
-0.784 \\
\end{array}$ & $\mathbf{B}^{A}$ & $\begin{array}{l}(S, 1) \\
0.945 \\
\end{array}$ & $\begin{array}{l}(2, L) \\
0.355 \\
\end{array}$ & $\mathbf{M d}^{A}$ & $\begin{array}{c}(1,2) \\
-0.375 \\
\end{array}$ & & & & & & & \\
\hline $\mathbf{M o}^{B}$ & $\begin{array}{l}(1,1) \\
0.328\end{array}$ & $\begin{array}{l}(1,2) \\
0.625\end{array}$ & $\begin{array}{l}(1,3) \\
0.115\end{array}$ & $\begin{array}{c}(2,2) \\
-0.045\end{array}$ & $\begin{array}{l}(2,3) \\
0.968\end{array}$ & $\begin{array}{c}(3,3) \\
-0.901\end{array}$ & $\mathbf{B}^{B}$ & $\begin{array}{l}(S, 1) \\
0.362\end{array}$ & $\begin{array}{l}(3, L) \\
0.486\end{array}$ & $\mathbf{M d} \mathbf{d}^{B}$ & $\begin{array}{r}(1,3 \\
-0.74\end{array}$ & & & & \\
\hline $\mathbf{M o}^{C}$ & $\begin{array}{l}(1,1) \\
0.218\end{array}$ & $\begin{array}{l}(1,2) \\
0.515\end{array}$ & $\begin{array}{c}(1,4) \\
-0.296\end{array}$ & $\begin{array}{l}(2,2) \\
0.334\end{array}$ & $\begin{array}{l}(2,3) \\
0.741\end{array}$ & $\begin{array}{l}(3,3) \\
0.304\end{array}$ & $\begin{array}{l}(3,4) \\
0.654\end{array}$ & $\begin{array}{l}(4,4) \\
0.376\end{array}$ & $\mathbf{B}^{C}$ & $\begin{array}{l}(S, 1) \\
0.238\end{array}$ & $\begin{array}{l}(4, L) \\
0.312\end{array}$ & $\mathbf{M} \mathbf{d}^{C}$ & $\begin{array}{l}(2,3) \\
0.312\end{array}$ & & \\
\hline $\mathbf{M o}^{D}$ & $\begin{array}{c}(1,1) \\
-0.398\end{array}$ & $\begin{array}{l}(1,2) \\
0.652\end{array}$ & $\begin{array}{c}(1,4) \\
-0.087\end{array}$ & $\begin{array}{l}(2,2) \\
0.499\end{array}$ & $\begin{array}{l}(2,3) \\
0.723\end{array}$ & $\begin{array}{l}(3,3) \\
0.568\end{array}$ & $\begin{array}{l}(3,4) \\
0.696\end{array}$ & $\begin{array}{l}(4,4) \\
0.004\end{array}$ & $\mathbf{B}^{D}$ & $\begin{array}{l}(S, 1) \\
0.311 \\
\end{array}$ & $\begin{array}{l}(4, L) \\
0.981 \\
\end{array}$ & $\mathbf{M d} \mathbf{d}^{D}$ & $\begin{array}{l}(2,3) \\
0.528\end{array}$ & $\begin{array}{c}(1,4) \\
-0.081\end{array}$ & \\
\hline Mo & $\begin{array}{c}(1,1) \\
-0.440\end{array}$ & $\begin{array}{l}(1,2) \\
0.804\end{array}$ & $\begin{array}{c}(2,2) \\
-0.239\end{array}$ & $\begin{array}{l}(2,3) \\
0.437\end{array}$ & $\begin{array}{l}(2,4) \\
0.035\end{array}$ & $\begin{array}{c}(3,3) \\
-0.045\end{array}$ & $\begin{array}{l}(3,4) \\
0.425\end{array}$ & $\begin{array}{l}(4,4) \\
0.002\end{array}$ & $\begin{array}{l}(4,5) \\
0.462\end{array}$ & $\begin{array}{r}(4,7) \\
-0.18\end{array}$ & $\begin{array}{l}5, \\
0.3\end{array}$ & $\begin{array}{l}5, \\
0.7\end{array}$ & $\begin{array}{l}(6,6) \\
0.304\end{array}$ & $\begin{array}{l}(6,7) \\
0.462\end{array}$ & $\begin{array}{c}(7,7) \\
-0.013\end{array}$ \\
\hline $\begin{array}{l}(7,8) \\
0.462\end{array}$ & $\begin{array}{c}(7,10) \\
-0.062\end{array}$ & $\begin{array}{l}(8,8) \\
0.499\end{array}$ & $\begin{array}{l}(8,9) \\
0.723\end{array}$ & $\begin{array}{l}(9,9) \\
0.568\end{array}$ & $\begin{array}{c}(9,10) \\
0.696\end{array}$ & $\begin{array}{c}(10,10) \\
0.004\end{array}$ & B & $\begin{array}{l}(S, 1) \\
0.945\end{array}$ & $\begin{array}{c}(10, L) \\
0.981\end{array}$ & Md & $\begin{array}{c}(1,2) \\
-0.268\end{array}$ & $\begin{array}{r}2,4 \\
-0.2\end{array}$ & $\begin{array}{l}(5,6) \\
0.312\end{array}$ & $\begin{array}{l}(8,9) \\
0.528\end{array}$ & $\begin{array}{c}(7,10) \\
-0.057\end{array}$ \\
\hline
\end{tabular}

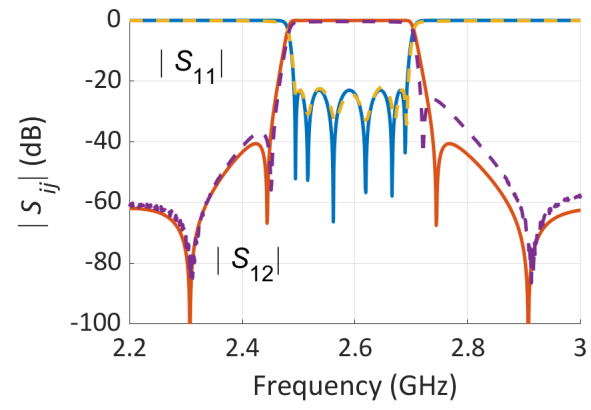

Fig. 13. Comparison between the measured response (dashed lines) and the synthesized response (solid lines).

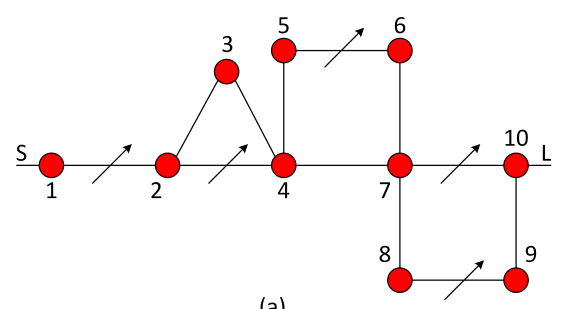

(a)

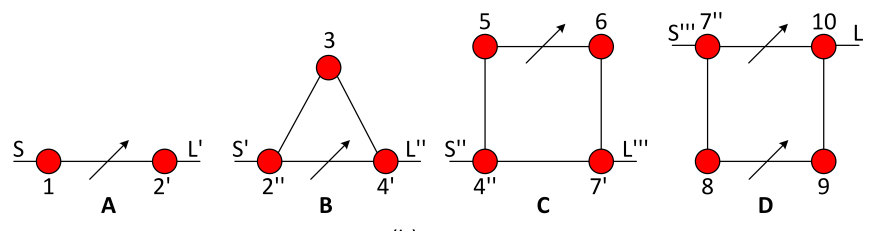

(b)

Fig. 14. (a) Topology of a ten-pole filter with the 4 dispersive blocks. (b) Separating the filter in (a) into 4 sub-filters labeled A, B, C, and D.

\section{B. Synthesis of a 10-Pole Dispersive Coupling Matrix}

In order do demonstrate the versatility and the robustness of the method we detail the synthesis of a 10-pole filter with $8 \mathrm{TZs}$ and a slightly more involved topology as depicted in Fig. 14 (a). As in the previous example, we first decompose the whole filter topology into 4 cascaded basic blocks shown in Fig. 14 (b). The TZ at $+3 j$ is assigned to the duplet $(1-2)$ (sub-filter A). Two complex conjugated TZs at $+0.1 j \pm 0.9$

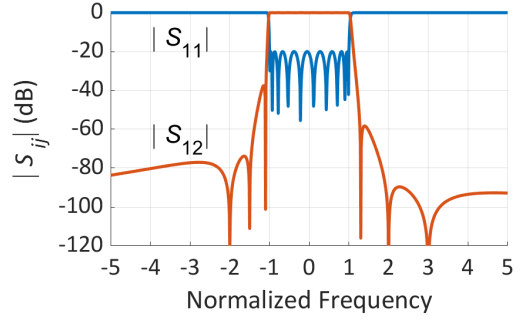

(a)

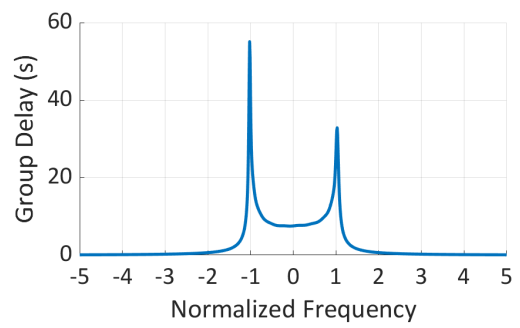

(b)

Fig. 15. (a) Response and (b) group delay of the synthesized dispersive coupling matrix (Md, Mo, B).

are assigned to the triplet $(2-3-4)$ (sub-filter B) to equalize the in-band group delay. Two asymmetric TZs at $+1.3 j$ and $-1.1 j$ are assigned to the quadruplet $(4-5-6-7)$ (subfilter C). Three TZs at $\pm 2 j$ and $-1.5 j$ are assigned to the other quadruplet $(7-8-9-10)$ (sub-filter D). The return loss level is designed to be $20 \mathrm{~dB}$.

The scattering matrix $S^{A}$ of sub-filter A can be obtained by extracting the corresponding TZs from the synthesized target matrix $S$ as

$$
\left(S^{A}, G^{A}\right)=\operatorname{Ext}(S,\{(+3 j, e),(\infty, p)\})
$$

with $G^{A}$ being the remaining scattering matrix. Then, scattering matrix $S^{B}$ of sub-filter B can be extracted from $G^{A}$ as

$$
\begin{aligned}
\left(S^{B}, G^{B}\right)=\operatorname{Ext}\left(G^{A},\{(+0.1 j+0.9, e),\right. \\
(+0.1 j-0.9, e),(\infty, p)\})
\end{aligned}
$$

Finally, by extracting $S^{C}$ from $G^{B}$, the scattering matrix $S^{D}$ 
of sub-filter $\mathrm{D}$ is obtained as the remaining response.

$\left(S^{C}, S^{D}\right)=\operatorname{Ext}\left(G^{B},\{(\infty, e),(+1.3 j, e),(-1.1 j, e),(\infty, p)\}\right)$

Starting from $S^{A}, S^{B}, S^{C}$ and $S^{D}$ canonical non-dispersive coupling matrices are computed, for example in transversal form. These coupling matrices are then further transformed to the targeted dispersive coupling matrices with specified topologies, the coupling coefficients of which are all listed in TABLE II. Eventually, the circuits A, B, C and D are cascaded together to yield the global dispersive coupling matrix of the 10-pole filter, whose coupling coefficients are listed in TABLE II. The response of this synthesized dispersive coupling matrix is shown in Fig. 15 (a) and can't be distinguished from $S$ in terms of modulus and group delay (see Fig. 15 (b)). This example further demonstrates the accuracy and flexibility of the proposed method when applied to high-order filters with a complex cascaded topology.

\section{CONCLUSION}

A general framework tailored to handle the synthesis of circuits with cascaded structures has been presented. One remarkable aspect of the associated decomposition procedure is that it takes place at the functional level of the filter's S-matrix: it provides therefore a quantitative assessment of the intuitive claim that in cascaded topologies each sub-circuit is tasked with generating and controlling a specific subset of the TZs. The ability to set the topology of each sub-block independently paves the way to hybrid implementations combining sections with cross-couplings, phase shifting (extracted pole), non-resonating nodes, and dispersive couplings. As for the dispersive coupling paradigm one can expect that with the provided shortest path rule and the direct synthesis by means of congruent transformations it will become a classic refinement of the coupled resonator model. We think in particular that its use should be considered in de-embedding techniques and Computer Aided Tuning (CAT) applications to take into account the inherent dispersive nature of couplings usually considered as a spurious phenomenon in current frequencyindependent approaches. In addition, extensions of analytical techniques to wide-band situations, as considered in [23] by means of optimisation techniques, and the associated CAT techniques tailored for this case [37], [38], look promising.

\section{APPENDIX}

We will not give detailed mathematical proofs of proposition 3.1 which are, for some, beyond the scope of this paper but merely sketch the underlying reasoning. In i) formula (19) is a compact form of the classical nodal equations used to compute the admittance response $Y$ of a low-pass circuit, and can be obtained by computing the Laplace transform of (18). ii) The realization can for example be obtained using the classical transversal form. The fact that congruent transformations leave the admittance matrix unchanged can be directly verified using the formulas in i). The converse is classical system theory, see for example [4], [31]. Eventually the shortest path rule comes from the formal expansion at $\infty$ of

$$
Y(s)=\sum_{k=1}^{\infty} \frac{G^{k} \stackrel{\text { def }}{=} B^{t}\left(\mathbf{M d}^{-1} \mathbf{M}_{0}\right)^{k-1} \mathbf{M d}^{-1} B}{s^{k}}
$$

There exists a fruitful link between matrices and graph theory, described for example in [39]. Using the classical association between a graph and its distance matrix, we first obtain a useful result relating the nullity of certain elements of $\mathbf{M d}^{-1}$ with properties of the graph associated to $\mathbf{M d}$ : if there is no path in this graph from node $k$ to node $l$ then $\left(\mathbf{M d}^{-1}\right)_{k, l}=0$. This property is obtained as a direct consequence of the Cayley-Hamilton theorem that allows to express $\mathbf{M d}^{-1}$ in terms of a polynomial in the matrix variable $\mathbf{M d}$. Then a careful study of the relations between the matrices $\mathbf{M d}^{-1}$, Mo and the introduced coupling graph yields the following: if there is no input to output path of length less than $m$ in the coupling graph then $\left(G^{m}\right)_{1,2}=0$ (the $2 \times 2$ matrices $G^{m}$, defined in (29), are the Markov parameters of the system [31]). Therefore, if $c$ is the length of the shortest path, we have that for $1 \leq k \leq c-1,\left(G^{k}\right)_{1,2}=0$ and hence the first non-vanishing term in the expansion of $Y_{1,2}$ is $\frac{\left(G^{c}\right)_{1,2}}{s^{l}}$. We conclude that at least $c-1 \mathrm{TZs}$ lie at infinity which was to be shown.

\section{REFERENCES}

[1] R. J. Cameron, C. M. Kudsia, and R. Mansour, Microwave Filters for Communication Systems: Fundamentals Design and Applications, 2nd ed. Hoboken, NJ, USA: Wiley, 2018.

[2] R. J. Cameron, A. R. Harish, and C. J. Radcliffe, "Synthesis of advanced microwave filters without diagonal cross-couplings," IEEE Trans. Microw. Theory Tech., vol. 50, no. 12, pp. 2862-2872, 2002.

[3] S. Tamiazzo and G. Macchiarella, "An analytical technique for the synthesis of cascaded n-tuplets cross-coupled resonators microwave filters using matrix rotations," IEEE Trans. Microw. Theory Tech., vol. 53, no. 5, pp. 1693-1698, 2005.

[4] F. Seyfert, "Analytical methods for the synthesis of microwave devices," professional thesis, Université Côte d'Azur, 2019, https://hal.inria.fr/tel02444432/document.

[5] S. Amari and G. Macchiarella, "Synthesis of inline filters with arbitrarily placed attenuation poles by using nonresonating nodes," IEEE Trans. Microw. Theory Tech., vol. 53, no. 10, pp. 3075-3081, 2005.

[6] M. Ohira, Z. Ma, H. Deguchi, and M. Tsuji, "A novel coaxial-excited fss-loaded waveguide filter with multiple transmission zeros," in 2010 Asia-Pacific Microw. Conf., 2010, pp. 1720-1723.

[7] U. Rosenberg and S. Amari, "A novel band-reject element for pseudoelliptic bandstop filters," IEEE Trans. Microw. Theory Tech., vol. 55, no. 4, pp. 742-746, 2007.

[8] M. Politi and A. Fossati, "Direct coupled waveguide filters with generalized chebyshev response by resonating coupling structures," in 40th Eur. Microw. Conf., 2010, pp. 966-969.

[9] U. Rosenberg, S. Amari, and F. Seyfert, "Pseudo-elliptic direct-coupled resonator filters based on transmission-zero-generating irises," in 40th Eur. Microw. Conf., 2010, pp. 962-965.

[10] S. Bastioli, R. V. Snyder, and P. Jojic, "High power in-line pseudoelliptic evanescent mode filter using series lumped capacitors," in 41st Eur. Microw. Conf., 2011, pp. 87-90.

[11] S. Amari, M. Bekheit, and F. Seyfert, "Notes on bandpass filters whose inter-resonator coupling coefficients are linear functions of frequency," in IEEE MTT-S Int. Microw. Symp. Dig, 2008, pp. 1207-1210.

[12] L. Szydlowski, A. Lamecki, and M. Mrozowski, "Coupled-resonator filters with frequency-dependent couplings: Coupling matrix synthesis," IEEE Microw. Wireless Compon. Lett., vol. 22, no. 6, pp. 312-314, 2012.

[13] L. Szydlowski, N. Leszczynska, and M. Mrozowski, "Dimensional synthesis of coupled-resonator pseudoelliptic microwave bandpass filters with constant and dispersive couplings," IEEE Trans. Microw. Theory Tech., vol. 62, no. 8, pp. 1634-1646, 2014. 
[14] L. Szydlowski, A. Lamecki, and M. Mrozowski, "A novel coupling matrix synthesis technique for generalized chebyshev filters with resonant source-load connection," IEEE Trans. Microw. Theory Tech., vol. 61, no. 10 , pp. $3568-3577,2013$

[15] Y. Zhang, H. Meng, and K.-L. Wu, "Synthesis of microwave filters with dispersive coupling using isospectral flow method," in IEEE MTT-S Int. Microw. Symp. Dig, 2019, pp. 846-848.

[16] Y. Zhang, K.-L. Wu, and F. Seyfert, "A preconditioner for synthesizing dispersive bandpass filters using isospectral flow method," in 2019 IEEE Asia-Pacific Microw. Conf., 2019, pp. 348-350.

[17] Y. Zhang, H. Meng, and K.-L. Wu, "Direct synthesis and design of dispersive waveguide bandpass filters," IEEE Trans. Microw. Theory Tech., vol. 68, no. 5, pp. 1678-1687, 2020.

[18] L. Szydlowski, N. Leszczynska, and M. Mrozowski, "Generalized chebyshev bandpass filters with frequency-dependent couplings based on stubs," IEEE Trans. Microw. Theory Tech., vol. 61, no. 10, pp. 3601$3612,2013$.

[19] S. Tamiazzo and G. Macchiarella, "Synthesis of cross-coupled filters with frequency-dependent couplings," IEEE Trans. Microw. Theory Tech., vol. 65, no. 3, pp. 775-782, 2017.

[20] Y. He, G. Macchiarella, G. Wang, W. Wu, L. Sun, L. Wang, and R. Zhang, "A direct matrix synthesis for in-line filters with transmission zeros generated by frequency-variant couplings," IEEE Trans. Microw. Theory Tech., vol. 66, no. 4, pp. 1780-1789, 2018.

[21] Y. He, G. Macchiarella, Z. Ma, L. Sun, and N. Yoshikawa, "Advanced direct synthesis approach for high selectivity in-line topology filters comprising $n-1$ adjacent frequency-variant couplings," IEEE Access, vol. 7, pp. 41 659-41668, 2019.

[22] P. Zhao and K. Wu, "Cascading fundamental building blocks with frequency-dependent couplings in microwave filters," IEEE Trans. Microw. Theory Tech., vol. 67, no. 4, pp. 1432-1440, 2019.

[23] W. Meng, H. Lee, K. A. Zaki, and A. E. Atia, "Synthesis of wideband multicoupled resonators filters," IEEE Trans. Microw. Theory Tech., vol. 59, no. 3, pp. 593-603, 2011.

[24] S. Amari, "On the maximum number of finite transmission zeros of coupled resonator filters with a given topology," IEEE Microw. Guided Wave Lett., vol. 9, no. 9, pp. 354-356, 1999.

[25] F. Seyfert and S. Bila, "General synthesis techniques for coupled resonator networks," IEEE Microw. Mag., vol. 8, no. 5, pp. 98-104, 2007.

[26] H. J. Carlin and P. P. Civalleri, Wideband Circuit Design, 1st ed. NY, USA: CRC Press, 1998.

[27] D. Pozar, Microwave Engineering, 4th Edition. Wiley, 2011.

[28] J. A. Ball, I. Gohberg, and L. Rodman, Interpolation of Rational Matrix Functions, ser. OT. Birkhäuser, 1990, vol. 44.

[29] J. O. Scanlan and J. D. Rhodes, "Unified theory of cascade synthesis," Proc. of the Institution of Elect. Engineers, vol. 117, no. 4, pp. 665-670, 1970.

[30] D. Youla, "A new theory of broad-band matching," IEEE Trans. Circuit Theory, vol. 11, no. 1, pp. 30-50, 1964.

[31] T. Kailath, Linear Systems, ser. Information and System Sciences Series. Prentice-Hall, 1980

[32] H. C. Bell, "Alternate symmetric coupled-resonator prototypes," in IEEE MTT-S Int. Microw. Symp. Dig, 2014, pp. 1-3.

[33] Xinshe Yin, "Accurate extraction of coupling matrix for coupled resonator filters," in IEEE MTT-S Int. Microw. Symp. Dig, 2012, pp. 1-3.

[34] J. Jia, Z. Zhang, C. Hu, Z. Liu, J. Yang, and Z. Shi, "Siw bandpass filters with frequency-dependent coupling for power wireless private network communication," in Photon. Electromagn. Res. Symp., 2019, pp. 30713075.

[35] L. Szydlowski, N. Leszczynska, A. Lamecki, and M. Mrozowski, "A substrate integrated waveguide (siw) bandpass filter in a box configuration with frequency-dependent coupling," IEEE Microw. Wireless Compon. Lett., vol. 22, no. 11, pp. 556-558, 2012.

[36] J.-M. Zhu, Y.-F. Xue, L. Sun, F. Liu, and H.-W. Deng, "Compact high-selectivity tunable dual-mode filter with constant bandwidth by adopting frequency-dependent s-1 coupling," Microw. and Opt. Technol. Lett., vol. 62, no. 1, pp. 108-111, 2020. [Online]. Available: https://onlinelibrary.wiley.com/doi/abs/10.1002/mop.32031

[37] H. Lee, K. A. Zaki, A. E. Atia, and A. J. Piloto, "Design and diagnosis of wideband coupled-resonator bandpass filters," IEEE Trans. Microw. Theory Tech., vol. 60, no. 5, pp. 1266-1277, 2012.

[38] H. Jia and R. R. Mansour, "An efficient technique for tuning and design of filters and diplexers," IEEE Trans. Microw. Theory Tech., vol. 68, no. 7, pp. 2610-2624, 2020.

[39] R. A. Brualdi and H. J. Ryser, Combinatorial Matrix Theory. Cambridge University Press, 1991.

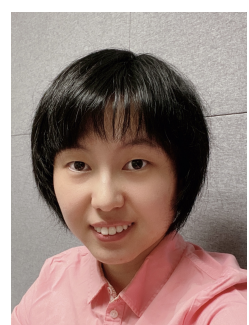

Yan Zhang (S'18) received the B.S. degree in electronic engineering from the University of Electronic Science and Technology of China, Chengdu, China, in 2017. She is currently pursuing the Ph.D. degree at the Chinese University of Hong Kong, Shatin, Hong Kong. Her current research interests include synthesis and tuning of filters with dispersive couplings and filers with irregular topologies.

Ms. Zhang is a recipient of Hong Kong Ph.D Fellowship.

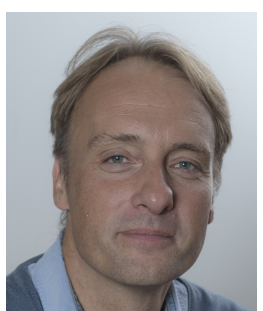

Fabien Seyfert graduated from the "Ecole superieure des Mines" (Engineering School) in St Etienne (France) in 1993 and received his Ph.D in mathematics in 1998. From 1998 to 2001 he joined Siemens (Munich, Germany) as a researcher specialized in discrete and continuous optimization methods. Since 2002 he occupies a full research position at INRIA (french agency for computer science and control, Nice, France). His research interest focuses on the conception of effective mathematical procedures and associated software for problems from signal processing including computer aided techniques for the design and tuning of microwave devices. In particular, he is the author of the software toolboxes Dedale-HF and Presto-HF.

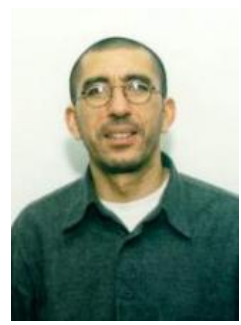

Smain Amari received the M.Eng. degree in electrical engineering and the $\mathrm{PhD}$ degree in theoretical physics from Washington University, St.Louis, in 1989 and 1994, respectively. He is presently professor of electrical and computer engineering at the Royal Military College, Kingston, Canada.

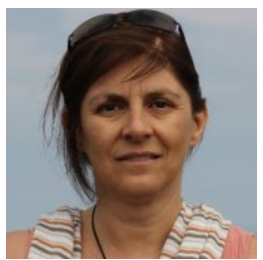

Martine Olivi was born in France, in 1958. She got the engineer's degree from Ecole des Mines de StEtienne, France, and the PhD degree in Mathematics from Université de Provence, Marseille, France, in 1983 and 1987 respectively. Since 1988, she is with the Institut National de Recherche en Informatique et Automatique (INRIA), Sophia Antipolis, France. Her research interests include: rational approximation, parametrization of linear multivariable systems, Schur analysis, identification and design of resonant systems.

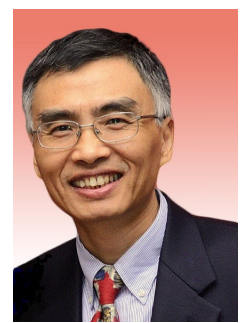

Ke-Li Wu (M'90-SM'96-F'11) received the B.S. and M.Eng. degrees from the Nanjing University of Science and Technology, Nanjing, China, in 1982 and 1985, respectively, and the Ph.D. degree from Laval University, Quebec, QC, Canada, in 1989. From 1989 to 1993, he was a Research Engineer with McMaster University, Canada. He joined COM DEV (now Honeywell Aerospace), Cambridge, ON Canada in 1993, where he was a Principal Member of Technical Staff. Since 1999, he has been with The Chinese University of Hong Kong, Hong Kong, where he is currently a Professor and the Director of the Radiofrequency Radiation Research Laboratory. His current research interests include EM-based circuit domain modeling of high-speed interconnections, robot automatic tuning of microwave filters, decoupling techniques of MIMO antennas, and IoT technologies. Prof. Wu is a member of the IEEE MTT-8 Subcommittee. He was a recipient of the 1998 COM DEV Achievement Award and the AsiaPacific Microwave Conference Prize twice in 2008 and 2012, respectively. $\mathrm{He}$ was an Associate Editor of the IEEE Transactions on MTT from 2006 to 2009. 